\title{
Sum of weighted Lebesgue spaces and nonlinear elliptic equations
}

\author{
Marino Badiale, Lorenzo Pisani, and Sergio Rolando
}

\begin{abstract}
We study the sum of weighted Lebesgue spaces, by considering an abstract measure space $(\Omega, \mathcal{A}, \mu)$ and investigating the main properties of both the Banach space

$L(\Omega)=\left\{u_{1}+u_{2}: u_{1} \in L^{q_{1}}(\Omega), u_{2} \in L^{q_{2}}(\Omega)\right\}, L^{q_{i}}(\Omega):=L^{q_{i}}(\Omega, d \mu)$, and the Nemytskil operator defined on it. Then we apply our general results to prove existence and multiplicity of solutions to a class of nonlinear $p$-Laplacian equations of the form

$$
-\triangle_{p} u+V(|x|)|u|^{p-2} u=f(|x|, u) \quad \text { in } \mathbb{R}^{N}
$$

where $V$ is a nonnegative measurable potential, possibly singular and vanishing at infinity, and $f$ is a Carathéodory function satisfying a double-power growth condition in $u$.
\end{abstract}

Mathematics Subject Classification (2010). Primary 35J62;

Secondary 46E30.

Keywords. Nonlinear elliptic equations, Zero mass case, Sum of weighted Lebesgue spaces, Nemytskiı̌ operator, Compact embeddings.

\section{Introduction}

The recent mathematical literature has seen a growing interest in what we may call, borrowing a terminology from [19], the zero mass case of noncritical elliptic problems of the form

$$
-\triangle u+V(x) u=g(u) \quad \text { in } \mathbb{R}^{N}, \quad N \geq 3,
$$

namely, the case of nonlinearities different from the critical power and such

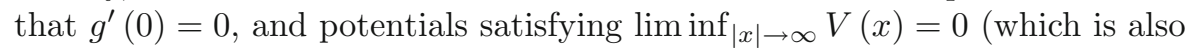
a particular critical frequency case, as termed in $[23,24])$.

Supported by MIUR, PRIN project 'Metodi variazionali e topologici nello studio di fenomeni non lineari'. 
These problems are variational in nature and the main difficulty in studying existence is that the related energy space

$$
H^{1}\left(\mathbb{R}^{N}, V\right):=\left\{u \in D^{1,2}\left(\mathbb{R}^{N}\right): \int_{\mathbb{R}^{N}} V(x) u^{2} d x<+\infty\right\}
$$

is not necessarily contained in any Lebesgue space $L^{q}\left(\mathbb{R}^{N}\right)$ with $q \neq 2^{*}:=$ $2 N /(N-2)$ and thus, not only the standard compact embeddings of $H^{1}\left(\mathbb{R}^{N}\right)$ are not available, but also single-power growth conditions of the form $|g(u)| \leq$ (const.) $|u|^{q}$ (with $q \neq 2^{*}$ ) do not ensure the finiteness of the energy functional of the equation on $H^{1}\left(\mathbb{R}^{N}, V\right)$.

The existence of solutions for a null potential $V=0$ was obtained by Berestycki and Lions in [19], where the authors probably first used the socalled double-power growth condition on $g$, namely, $g(u)$ behaves as a subcritical power $u^{q_{1}-1}$ at infinity and a supercritical power $u^{q_{2}-1}$ near the origin, where $q_{1}<2^{*}<q_{2}$. A multiplicity result was also obtained in [20].

More recently, the zero mass case of equations (1.1) with noncritical nonlinearities behaving as a single power has been widely studied in both the autonomous and nonautonomous cases (see e.g. [10,12,22, 25,26,37] and $[1,3,21,31,37]$ respectively, and the references therein), showing essentially that the existence of solutions relies on suitable compatibility conditions between the power of $u$ and the growth and decaying rates of $V(x)$ (and possibly of the nonlinearity) at zero and infinity.

Besides, many authors resumed the study of equation (1.1) under the double-power growth condition, after it was successfully exploited in $[14,15]$ in dealing with the semilinear Maxwell equations (see also $[4,5]$ for other recent works using the double-power assumption). The autonomous zero mass case of (1.1) has been considered, e.g., in [7-9,11,13,16-18], where it is seen that the double-power assumption allows the potential $V$ to be very general and no compatibility condition is needed in order to get existence. For instance, the radial existence and multiplicity results of [11] only require the mild integrability assumption $(\mathbf{V})$ below, in such a way that no behaviour is prescribed either at infinity or at the origin to the potential, which may also have a nonempty, even continuous, set of singularities. As far as we know, the nonautonomous zero mass case of equation (1.1) is only studied in [6], where (1.1) is considered with $V=0$ and $g(x, u)=K(x) f(u)$, and in [28], where the authors assume $V<0$ and deal with nonlinearities of the form $g(x, u)=f(u)+K(x)$.

Here we study the nonautonomous radial case of equation (1.1) with nonnegative potentials and double-power nonlinearities, by actually considering the more general $p$-Laplacian problem

$$
-\triangle_{p} u+V(|x|)|u|^{p-2} u=f(|x|, u) \quad \text { in } \mathbb{R}^{N}
$$

where $1<p<N$ and $\triangle_{p} u=\operatorname{div}\left(|\nabla u|^{p-2} \nabla u\right)$. More precisely, we assume that $V:(0,+\infty) \rightarrow[0,+\infty]$ and $f:(0,+\infty) \times \mathbb{R} \rightarrow[0,+\infty)$ are, respectively, a measurable and a Carathéodory function, both nonnegative; then we define 
the space

$$
W^{1, p}\left(\mathbb{R}^{N}, V\right):=\left\{u \in D^{1, p}\left(\mathbb{R}^{N}\right): \int_{\mathbb{R}^{N}} V(|x|)|u|^{p} d x<+\infty\right\}
$$

and prove existence and multiplicity of solutions to (1.2) in the following weak sense: we say that $u \in W^{1, p}\left(\mathbb{R}^{N}, V\right)$ is a weak solution to equation (1.2) if and only if

$$
\int_{\mathbb{R}^{N}}\left(|\nabla u|^{p-2} \nabla u \cdot \nabla h+V(|x|)|u|^{p-2} u h\right) d x=\int_{\mathbb{R}^{N}} f(|x|, u) h d x
$$

for all $h \in W^{1, p}\left(\mathbb{R}^{N}, V\right)$.

Denoting $F(x, t):=\int_{0}^{t} f(x, s) d s$ and $p^{*}:=p N /(N-p)$, we will exploit the following hypotheses, where (f) is a double-power growth condition:

(V) $V \in L^{1}((a, b))$ for some open bounded interval $(a, b)$ with $b>a>0$;

(f) there exist $\gamma>p$ such that for almost every $r>0$ and all $t \geq 0$ one has

$$
0 \leq \gamma F(r, t) \leq f(r, t) t \leq M \max \left\{r^{\theta_{1}}, r^{\theta_{2}}\right\} \min \left\{t^{q_{1}}, t^{q_{2}}\right\}
$$

for some constant $M>0$ and $\theta_{1}, \theta_{2}, q_{1}, q_{2} \in \mathbb{R}$ such that

$$
p<q_{1}<p^{*}+\frac{\theta_{1} p}{N-p} \leq p^{*}+\frac{\theta_{2} p}{N-p}<q_{2} .
$$

Note that (1.4) implies $f(\cdot, 0)=0$ almost everywhere and observe that the inequality $p<p^{*}+\frac{\theta_{1} p}{N-p} \leq p^{*}+\frac{\theta_{2} p}{N-p}$ of (1.5) is equivalent to

$$
\theta_{2} \geq \theta_{1}>-p
$$

The requirement $q_{1}>p$ is not restrictive and may also be avoided in (1.5).

Our existence and multiplicity results are the following Theorems 1.1 and 1.2 , which, as far as we know, are also new for the nonautonomous semilinear case $p=2$. Note that no assumptions neither on the regularity of $V$ nor on its behaviour at zero or infinity are made, and that $V=0$ is allowed in both Theorems 1.1 and 1.2.

Theorem 1.1. Assume $(\mathbf{V})$ and $(\mathbf{f})$. If there exists $t_{*}>0$ such that

$$
F(r, t)>0 \text { for almost every } r>0 \text { and all } t \geq t_{*},
$$

then equation (1.2) has a nontrivial nonnegative radial weak solution.

Theorem 1.2. Assume (V) and (f). If for almost every $r>0$ and all $t \geq 0$ one has

$$
\begin{gathered}
f(r, t)=-f(r,-t), \\
F(r, t) \geq m \max \left\{r^{\theta_{1}}, r^{\theta_{2}}\right\} \min \left\{t^{q_{1}}, t^{q_{2}}\right\}
\end{gathered}
$$

for some constant $m>0$ (with the same exponents $\theta_{1}, \theta_{2}, q_{1}, q_{2}$ of assumption (f)), then equation (1.2) has infinitely many radial weak solutions.

For instance, the assumptions of both Theorems 1.1 and 1.2 hold true for

$$
f(|x|, u)=|x|^{\theta} \frac{|u|^{q_{2}-2} u}{1+|u|^{q_{2}-q_{1}}} \quad \text { with } \theta>-p \text { and } q_{1}<p^{*}+\frac{\theta p}{N-p}<q_{2} \text {. }
$$


More generally, Theorem 1.1 applies for example to nonlinearities of the form

$$
f(|x|, u)=K(|x|) g(u)
$$

provided that $K:(0,+\infty) \rightarrow(0,+\infty)$ is measurable and such that limsup $\frac{K(r)}{r^{\theta_{1}}}<+\infty, \limsup _{r \rightarrow+\infty} \frac{K(r)}{r^{\theta_{2}}}<+\infty$ for some $\theta_{1}, \theta_{2}$ satisfying $(1.6)$, and $g: \begin{array}{r}r \rightarrow 0^{+} \\ : \mathbb{R} \rightarrow\end{array}$ $[0,+\infty)$ is continuous and satisfying $\limsup _{t \rightarrow 0^{+}} \frac{g(t)}{t^{q_{2}-1}}<+\infty, \limsup _{t \rightarrow+\infty} \frac{g(t)}{t^{q_{1}}-1}<+\infty$ for some $q_{1}, q_{2}$ as in (1.5) together with

$$
\int_{0}^{t_{*}} g(s) d s>0 \text { for some } t_{*}>0
$$

and the so-called Ambrosetti-Rabinowitz condition:

$$
\exists \gamma>p, \quad \forall t \geq 0, \quad \gamma \int_{0}^{t} g(s) d s \leq g(t) t .
$$

Theorems 1.1 and 1.2 will be proved in Sect. 5 by applying some abstract results from the previous sections, where we study of the sum $L^{q_{1}}+L^{q_{2}}$ of Lebesgue spaces $L^{q_{i}}$. In fact, for a complete treatment, variational problems involving double-power nonlinearities need the use of such a particular type of functional framework, to such an extent that its main properties have been investigated by different authors in different works, such as $[7,11,13,15,18,35]$ and the unpublished note [33], which is a preliminary version of our Sects. 2 and 3. These studies, though occasional, have clarified the main features of the $L^{q_{1}}+L^{q_{2}}$ spaces, showing essentially that they share several important properties with the usual Lebesgue spaces and that, in many respects, they play for the Sobolev space $D^{1,2}\left(\mathbb{R}^{N}\right)$ the same role that the usual Lebesgue spaces play for $H^{1}\left(\mathbb{R}^{N}\right.$ ) (see for instance [15, Lemma 3] and the compactness results of [7]). Here, owing to nonautonomous nonlinearities, we need to consider the sum of weighted Lebesgue spaces instead of the sum of the usual ones, so that the interest of our Sects. 2, 3 and 4 is actually twofold: on the one hand, we collect some general results which have already been used in many of the above-mentioned papers, but which are presently expounded in the unpublished works $[33,35]$ only; on the other, we extend the investigation to the sum of weighted Lebesgue spaces, by considering an abstract measure space $(\Omega, \mathcal{A}, \mu)$ and studying the space

$$
L(\Omega):=\left\{u_{1}+u_{2}: u_{1} \in L^{q_{1}}(\Omega), u_{2} \in L^{q_{2}}(\Omega)\right\}, \quad L^{q_{i}}(\Omega):=L^{q_{i}}(\Omega, d \mu) .
$$

Going into more detail, the paper is organized as follows.

Section 2 is devoted to the study of the basic properties of $L(\Omega)$. Some characterizations of the set $L(\Omega)$ are given in Proposition 2.3, showing in particular that it is made up by the measurable functions which are $q_{1}$-integrable on some measurable subset and $q_{2}$-integrable on its complement. Then we structure $L(\Omega)$ as a Banach space by introducing a natural family of equivalent norms and proving an isometrical identification between $L(\Omega)$ and the dual space of $L^{q_{1}^{\prime}}(\Omega) \cap L^{q_{2}^{\prime}}(\Omega), q_{i}^{\prime}=q_{i} /\left(q_{i}-1\right)$, endowed with the norm $\|\cdot\|_{L^{q_{1}^{\prime}}}$ 
$+\|\cdot\|_{L^{q_{2}^{\prime}}}$. The related topology is briefly studied and some useful inequalities are given in subsect. 2.3. In the last part of Sect. 2, we show that $L(\Omega)$ can be characterized as an Orlicz space (cf. [29,30,34]), whose Orlicz norm induces the same topology. We observe that some of the results of Sect. 2 could be deduced from the theory presented in [30, Chapter 12] for the sum of Orlicz spaces; nevertheless, the proofs are often simple and direct, so we give them anyway here, for sake of completeness.

The aim of Sect. 3 is the investigation of the Nemytskil operator $\mathcal{N}$ defined on $L(\Omega)$, which is a central topic in nonlinear analysis. Unfortunately, the characterization of $L(\Omega)$ as an Orlicz space is not very helpful in this direction, since, at least to our knowledge, not many results on the subject are available in the literature; for instance, the classical monograph [29] only gives some results for Orlicz spaces on a base set of finite measure, while the Nemytskil operator is considered in [27] just from the point of view of its good definition (see Remark 3.6 below). On the other hand, the case

$$
\mathcal{N}: L(\Omega) \rightarrow L^{q}(\Omega)
$$

can be studied through direct arguments, and we will show that, in this respect, $L(\Omega)$ behaves exactly as the usual Lebesgue spaces (cf. [38, Theorem 19.1]): whenever the Nemytskil operator is well defined, it is continuous and (under a suitable continuity assumption on the measure $\mu$ ) it is also bounded (see Theorems 3.1 and 3.4 for precise statements). A differentiability result will also be given (Proposition 3.8).

In Sect. 4 we prove a new compactness result (Theorem 4.1) involving $L(\Omega)$ with $\Omega=\mathbb{R}^{N}$ and $d \mu=\omega(x) d x$. Some consequences are pointed out at the end of the section (Corollaries 4.5 and 4.6), recovering a compactness lemma of [15] as a particular case.

Section 5 is finally devoted to the proofs of Theorems 1.1 and 1.2 .

Notations. We conclude this introductory section by defining some notations of frequent use throughout the paper.

- $\mathbb{N}$ is the set of natural numbers, including 0 .

- For every $r>0$, we set $B_{r}:=\left\{x \in \mathbb{R}^{N}:|x|<r\right\}$.

- For any subset $E$ of an ambient set $\Omega$ (which will be understood from the context), we set $E^{c}:=\Omega \backslash E$ and denote the characteristic function of $E$ by $\chi_{E}$.

- $\|\cdot\|_{X}$ and $X^{\prime}$ denote a norm and the dual space of a Banach space $X$, in which $\rightarrow$ and $\rightarrow$ mean strong and weak convergence respectively.

- $\hookrightarrow$ denotes continuous embeddings.

- $p^{\prime}:=p /(p-1)$ is the Hölder-conjugate exponent of $p$.

- $p^{*}:=p N /(N-p)$ is the Sobolev exponent related to $p$.

- For any map $u: \Omega \rightarrow \mathbb{R}$, we denote $\Lambda_{u}:=\Lambda_{u}(\Omega):=\{x \in \Omega:|u(x)|>1\}$.

\section{The space $L(\Omega)$}

Let $(\Omega, \mathcal{A}, \mu)$ be a nonempty $\sigma$-finite measure space and fix $1<q_{1} \leq q_{2}<\infty$. 
For any measurable set $E \subseteq \Omega$, we will omit the indication of the measure $\mu$ in the Lebesgue space notation $L^{p}(E, d \mu)$, simply writing $L^{p}(E)=$ $L^{p}(E, d \mu)$.

\subsection{Definitions and basic properties of $L(\Omega)$}

We denote by $\mathcal{M}(\Omega)$ the linear space of the real valued measurable functions defined on $\Omega$, in which equality is meant in the $\mu$-a.e. sense, and, as in (1.10), we define

$$
\begin{aligned}
L(\Omega) & :=L^{q_{1}}(\Omega)+L^{q_{2}}(\Omega) \\
& :=\left\{u \in \mathcal{M}(\Omega): u=u_{1}+u_{2}, u_{1} \in L^{q_{1}}(\Omega), u_{2} \in L^{q_{2}}(\Omega)\right\},
\end{aligned}
$$

which clearly contains both $L^{q_{1}}(\Omega)$ and $L^{q_{2}}(\Omega)$. Observe that the set $L(\Omega)$ is actually of interest only if $q_{1}<q_{2}$ and the base set $\Omega$ has infinite measure, since $\mu(\Omega)<+\infty$ or $q_{1}=q_{2}$ implies $L^{q_{2}}(\Omega) \subseteq L^{q_{1}}(\Omega)$ and thus $L(\Omega)=L^{q_{1}}(\Omega)$. Nevertheless, we do not require such restrictions, for future convenience in encompassing particular cases.

Proposition 2.1. Let $u \in L(\Omega)$ and let $E \subseteq \Omega$ be a measurable set. Then

$$
\mu(E)<+\infty \Rightarrow u \in L^{q_{1}}(E) \text { and } u \in L^{\infty}(E) \Rightarrow u \in L^{q_{2}}(E) .
$$

Proof. Let $u_{1} \in L^{q_{1}}(\Omega)$ and $u_{2} \in L^{q_{2}}(\Omega)$ be such that $u=u_{1}+u_{2}$. Then $\mu(E)<+\infty$ implies $L^{q_{2}}(E) \subseteq L^{q_{1}}(E)$ and thus $u=u_{1}+u_{2} \in L^{q_{1}}(E)$. Now assume $u \in L^{\infty}(E)$. Since $u_{1} \in L^{q_{1}}(E)$ and $u_{2} \in L^{q_{2}}(E)$, in order to get $u \in L^{q_{2}}(E)$ we need only to show that $u_{1} \in L^{q_{2}}(E)$. Setting $\Lambda_{u_{1}}=$ $\left\{x \in \Omega:\left|u_{1}(x)\right|>1\right\}$, we write

$$
E=\left(E \cap \Lambda_{u_{1}}\right) \cup\left(E \cap \Lambda_{u_{1}}^{c}\right)
$$

Since $q_{1}<q_{2}$ and $\left|u_{1}\right| \leq 1 \mu$-a.e. in $\Lambda_{u_{1}}^{c}$, we get

$$
\int_{E \cap \Lambda_{u_{1}}^{c}}\left|u_{1}\right|^{q_{2}} d \mu=\int_{E \cap \Lambda_{u_{1}}^{c}}\left|u_{1}\right|^{q_{2}-q_{1}}\left|u_{1}\right|^{q_{1}} d \mu \leq \int_{E \cap \Lambda_{u_{1}}^{c}}\left|u_{1}\right|^{q_{1}} d \mu<+\infty .
$$

On the other hand, one has

$$
\left|u_{1}\right|=\left|u-u_{2}\right| \leq\|u\|_{L^{\infty}(E)}+\left|u_{2}\right| \quad \mu \text {-a.e. in } E
$$

so that

$$
\begin{aligned}
\int_{E \cap \Lambda_{u_{1}}}\left|u_{1}\right|^{q_{2}} d \mu & \leq 2^{q_{2}-1} \int_{E \cap \Lambda_{u_{1}}}\left(\|u\|_{L^{\infty}(E)}^{q_{2}}+\left|u_{2}\right|^{q_{2}}\right) d \mu \\
& \leq 2^{q_{2}-1}\left(\|u\|_{L^{\infty}(E)}^{q_{2}} \mu\left(\Lambda_{u_{1}}\right)+\int_{E \cap \Lambda_{u_{1}}}\left|u_{2}\right|^{q_{2}} d \mu\right)<+\infty
\end{aligned}
$$

since $\Lambda_{u_{1}}$ has finite measure. 
Corollary 2.2. One has $L(\Omega) \cap L^{\infty}(\Omega)=L^{q_{2}}(\Omega) \cap L^{\infty}(\Omega)$.

Proof. Since the inclusion $L^{q_{2}}(\Omega) \cap L^{\infty}(\Omega) \subseteq L(\Omega) \cap L^{\infty}(\Omega)$ is obvious, the claim readily follows from Proposition 2.1.

We now give some first characterizations of the functions in $L(\Omega)$. In particular, we show that $L(\Omega)$ is the set of the functions $u \in \mathcal{M}(\Omega)$ which are $q_{1}$-integrable on some measurable subset $E \subseteq \Omega$ (possibly depending on $u$ ) and $q_{2}$-integrable on its complement $E^{c}$. For any $u \in L(\Omega)$, one of such subsets is

$$
\Lambda_{u}:=\Lambda_{u}(\Omega):=\{x \in \Omega:|u(x)|>1\},
$$

which is defined (up to null measure sets) for every $u \in \mathcal{M}(\Omega)$ and will play an important role hereafter.

Proposition 2.3. For any $u \in \mathcal{M}(\Omega)$, the following propositions are equivalent:

(i) $u \in L(\Omega)$

(ii) $u \in L^{q_{1}}(E) \cap L^{q_{2}}\left(E^{c}\right)$ for some measurable subset $E \subseteq \Omega$

(iii) $u \in L^{q_{1}}\left(\Lambda_{u}\right) \cap L^{q_{2}}\left(\Lambda_{u}^{c}\right)$ and $\mu\left(\Lambda_{u}\right)<+\infty$

(iv) $|u| \in L(\Omega)$

(v) $|u| \leq v$ for some $v \in L(\Omega)$.

Proof. Since the implications $\mathbf{i i i} \Rightarrow \mathbf{i i}$ and $\mathbf{i v} \Rightarrow \mathbf{v}$ are obvious, we need only to show that $\mathbf{i} \Rightarrow \mathbf{i i i}$, ii $\Rightarrow \mathbf{i}, \mathbf{i} \Rightarrow \mathbf{i v}$ and $\mathbf{v} \Rightarrow \mathbf{i}$.

$\left(\mathbf{i} \Rightarrow\right.$ iii) If $u \in L(\Omega)$ then $u \in L^{q_{2}}\left(\Lambda_{u}^{c}\right)$ by Proposition 2.1 and $u=u_{1}+u_{2}$ for some $u_{1} \in L^{q_{1}}(\Omega)$ and $u_{2} \in L^{q_{2}}(\Omega)$ by definition. Since $1<|u| \leq\left|u_{1}\right|+\left|u_{2}\right|$ implies $\left|u_{j}\right| \geq 1 / 2$ for some $j \in\{1,2\}$, we get

$$
+\infty>\int_{\Omega}\left|u_{j}\right|^{q_{j}} d \mu \geq \int_{\Lambda_{u}}\left|u_{j}\right|^{q_{j}} d \mu \geq \frac{1}{2^{q_{j}}} \int_{\Lambda_{u}} d \mu=\frac{1}{2^{q_{j}}} \mu\left(\Lambda_{u}\right),
$$

which also yields that $u \in L^{q_{1}}\left(\Lambda_{u}\right)$ by Proposition 2.1 again.

$(\mathbf{i i} \Rightarrow \mathbf{i})$ If $u \in L^{q_{1}}(E) \cap L^{q_{2}}\left(E^{c}\right)$ for some measurable set $E \subseteq \Omega$ then $u=u \chi_{E}+u \chi_{E^{c}}$ with $u \chi_{E} \in L^{q_{1}}(\Omega)$ and $u \chi_{E^{c}} \in L^{q_{2}}\left(E^{c}\right)$, which means $u \in L(\Omega)$.

(i $\Rightarrow \mathbf{i v ) ~ I t ~ f o l l o w s ~ f r o m ~ f r o m ~ t h e ~ a l r e a d y ~ p r o v e d ~ e q u i v a l e n c e ~} \mathbf{i} \Leftrightarrow$ iii , since $u \in L^{q_{1}}\left(\Lambda_{u}\right) \cap L^{q_{2}}\left(\Lambda_{u}^{c}\right) \Leftrightarrow|u| \in L^{q_{1}}\left(\Lambda_{u}\right) \cap L^{q_{2}}\left(\Lambda_{u}^{c}\right)$ and $\Lambda_{u}=\Lambda_{|u|}$.

$(\mathbf{v} \Rightarrow \mathbf{i})$ If $v \in L(\Omega)$ then $v \in L^{q_{1}}\left(\Lambda_{v}\right) \cap L^{q_{2}}\left(\Lambda_{v}^{c}\right)$ by implication $\mathbf{i} \Rightarrow$ iii, so that $|u| \leq v$ implies $u \in L^{q_{1}}\left(\Lambda_{v}\right) \cap L^{q_{2}}\left(\Lambda_{v}^{c}\right)$. This gives that $u \in L(\Omega)$ since ii $\Rightarrow$ i.

\subsection{The Banach structure of $L(\Omega)$}

The set $L(\Omega)$ has a natural linear structure as a subspace of $\mathcal{M}(\Omega)$ and can be equipped with a family of equivalent norms by setting

$$
\|u\|:=\|u\|_{1}:=\inf _{u_{1}+u_{2}=u}\left(\left\|u_{1}\right\|_{L^{q_{1}}(\Omega)}+\left\|u_{2}\right\|_{L^{q_{2}(\Omega)}}\right)
$$




$$
\begin{gathered}
\|u\|_{t}:=\inf _{u_{1}+u_{2}=u}\left(\left\|u_{1}\right\|_{L^{q_{1}}(\Omega)}^{t}+\left\|u_{2}\right\|_{L^{q_{2}}(\Omega)}^{t}\right)^{1 / t} \text { for } 1<t<\infty \\
\|u\|^{*}:=\|u\|_{\infty}:=\inf _{u_{1}+u_{2}=u} \max \left\{\left\|u_{1}\right\|_{L^{q_{1}(\Omega)}},\left\|u_{2}\right\|_{L^{q_{2}(\Omega)}}\right\} .
\end{gathered}
$$

Notice that

$$
\|u\|_{t}=\inf _{u_{1}+u_{2}=u}\left\|\left(u_{1}, u_{2}\right)\right\|_{t} \quad \text { for every } 1 \leq t \leq \infty
$$

where

$$
\left\|\left(u_{1}, u_{2}\right)\right\|_{t}:= \begin{cases}\left(\left\|u_{1}\right\|_{L^{q_{1}}(\Omega)}^{t}+\left\|u_{2}\right\|_{L^{q_{2}}(\Omega)}^{t}\right)^{1 / t} & \text { if } 1 \leq t<\infty \\ \max \left\{\left\|u_{1}\right\|_{L^{q_{1}}(\Omega)},\left\|u_{2}\right\|_{L^{q_{2}}(\Omega)}\right\} & \text { if } t=\infty\end{cases}
$$

defines the usual family of equivalent norms of $L^{q_{1}}(\Omega) \times L^{q_{2}}(\Omega)$.

Proposition 2.4. $\left\{\|\cdot\|_{t}\right\}_{1 \leq t \leq \infty}$ is a family of equivalent norms on $L(\Omega)$. Moreover, $\|u\|_{t}=\||u|\|_{t}$ for every $u \in L(\Omega)$ and $1 \leq t \leq \infty$.

Proof. The fact that the functionals (2.2)-(2.4) define a family of norms of $L(\Omega)$ is trivial. We just observe that such functionals are positive definite since $\|u\|_{t}=0$ implies the existence of $\left(u_{1, n}, u_{2, n}\right) \in L^{q_{1}}(\Omega) \times L^{q_{2}}(\Omega)$ such that $u=u_{1, n}+u_{2, n} \rightarrow 0 \mu$-a.e. in $\Omega$ as $n \rightarrow \infty$. The equivalence readily follows from the fact that the right hand sides of $(2.2)-(2.4)$ are the infima of equivalent norms of $L^{q_{1}}(\Omega) \times L^{q_{2}}(\Omega)$. Finally, if $u \in L(\Omega), u_{1} \in L^{q_{1}}(\Omega)$, $u_{2} \in L^{q_{2}}(\Omega)$ are such that $u=u_{1}+u_{2}$, letting $\operatorname{sign}(u):=\chi_{\{u>0\}}-\chi_{\{u<0\}}$ we have $|u|=\operatorname{sign}(u) u=\operatorname{sign}(u) u_{1}+\operatorname{sign}(u) u_{2}$ with $\left\|\operatorname{sign}(u) u_{1}\right\|_{L^{q_{1}(\Omega)}}=$ $\left\|u_{1}\right\|_{L^{q_{1}}(\Omega)}$ and $\left\|\operatorname{sign}(u) u_{2}\right\|_{L^{q_{2}(\Omega)}}=\left\|u_{2}\right\|_{L^{q_{2}(\Omega)}}$, which implies $\||u|\|_{t} \leq\|u\|_{t}$. Similarly, if $\bar{u}_{1} \in L^{q_{1}}(\Omega)$ and $\bar{u}_{2} \in L^{q_{2}}(\Omega)$ are such that $|u|=\bar{u}_{1}+\bar{u}_{2}$, then $u=\operatorname{sign}(u)|u|=\operatorname{sign}(u) \bar{u}_{1}+\operatorname{sign}(u) \bar{u}_{2}$ yields $\|u\|_{t} \leq\||u|\|_{t}$.

Proposition 2.5. The infimum in $\|\cdot\|_{t}$ is attained for every $1 \leq t \leq \infty$.

Proof. Let $1 \leq t \leq \infty$, fix $u \in L(\Omega)$ and consider a minimizing sequence for $\|u\|_{t}$, namely $\left\|\left(u_{1, n}, u_{2, n}\right)\right\|_{t} \rightarrow\|u\|_{t}$ and $u=u_{1, n}+u_{2, n} \mu$-a.e. in $\Omega$. Since $L^{q_{1}}(\Omega) \times L^{q_{2}}(\Omega)$ is a reflexive Banach space with respect to the norm $(2.5)$, up to a subsequence there exists $\left(u_{1}, u_{2}\right) \in L^{q_{1}}(\Omega) \times L^{q_{2}}(\Omega)$ such that $\left(u_{1, n}, u_{2, n}\right) \rightarrow\left(u_{1}, u_{2}\right)$ in $L^{q_{1}}(\Omega) \times L^{q_{2}}(\Omega)$ and

$$
\left\|\left(u_{1}, u_{2}\right)\right\|_{t} \leq \liminf _{n \rightarrow \infty}\left\|\left(u_{1, n}, u_{2, n}\right)\right\|_{t}=\|u\|_{t} .
$$

Now we observe that the linear mapping defined by $(v, w) \mapsto v+w$ is continuous from $L^{q_{1}}(\Omega) \times L^{q_{2}}(\Omega)$ into $L(\Omega)$, as one has

$$
\|v+w\|_{t} \leq\|v\|_{t}+\|w\|_{t} \leq\|v\|_{L^{q_{1}}(\Omega)}+\|w\|_{L^{q_{2}(\Omega)}}=\|(v, w)\|_{1} .
$$

Hence $u_{1, n}+u_{2, n} \rightarrow u_{1}+u_{2}$ in $L(\Omega)$. Therefore, $u_{1, n}+u_{2, n}=u$ implies $u=u_{1}+u_{2}$ by uniqueness of the weak limit, so that $\|u\|_{t} \leq\left\|\left(u_{1}, u_{2}\right)\right\|_{t}$ by definition of $\|u\|_{t}$. Together with (2.6), this means $\|u\|_{t}=\left\|\left(u_{1}, u_{2}\right)\right\|_{t}$.

Proposition 2.6. The norm $\|\cdot\|_{t}$ is uniformly convex for $1<t<\infty$. 
Proof. Let $1<t<\infty$. From the abstract theory of product spaces, the norm (2.5) is uniformly convex, that is, $\forall \varepsilon>0$ there exists $\delta>0$ such that

$$
\left.\begin{array}{l}
\left\|\left(u_{1}, u_{2}\right)\right\|_{t},\left\|\left(v_{1}, v_{2}\right)\right\|_{t} \leq 1 \\
\left\|\left(u_{1}-v_{1}, u_{2}-v_{2}\right)\right\|_{t}>\varepsilon
\end{array}\right\} \Rightarrow \frac{1}{2}\left\|\left(u_{1}+v_{1}, u_{2}+v_{2}\right)\right\|_{t}<1-\delta .
$$

Now, if $u, v \in L(\Omega)$ satisfy $\|u\|_{t} \leq 1,\|v\|_{t} \leq 1$ and $\|u-v\|_{t}>\varepsilon$, then by Proposition 2.5 there exist $\left(u_{1}, u_{2}\right),\left(v_{1}, v_{2}\right) \in L^{q_{1}}(\Omega) \times L^{q_{2}}(\Omega)$ such that $\left\|\left(u_{1}, u_{2}\right)\right\|_{t}=\|u\|_{t} \leq 1,\left\|\left(v_{1}, v_{2}\right)\right\|_{t}=\|v\|_{t} \leq 1$ and $\varepsilon<\|u-v\|_{t} \leq \|\left(u_{1}-v_{1}\right.$, $\left.u_{2}-v_{2}\right) \|_{t}$. Hence $\|u+v\|_{t} \leq\left\|\left(u_{1}+v_{1}, u_{2}+v_{2}\right)\right\|_{t}<2(1-\delta)$.

According to Proposition 2.4, we will henceforth consider $L(\Omega)$ as a normed topological vector space, equipped with the equivalent norms (2.2)-(2.4).

Proposition 2.7. Let $\left\{u_{n}\right\} \subseteq L(\Omega)$ be such that for every $\varepsilon>0$ there exist $n_{\varepsilon}>0$ and a sequence of measurable sets $E_{\varepsilon, n} \subseteq \Omega$ satisfying

$$
\forall n>n_{\varepsilon}, \quad \int_{E_{\varepsilon, n}}\left|u_{n}\right|^{q_{1}} d \mu+\int_{E_{\varepsilon, n}^{c}}\left|u_{n}\right|^{q_{2}} d \mu<\varepsilon .
$$

Then $u_{n} \rightarrow 0$ in $L(\Omega)$.

Proof. Let $E=E_{\varepsilon, n}$ for brevity. Since $u_{n}=u_{n} \chi_{E}+u_{n} \chi_{E^{c}}$, (2.7) implies $u_{n} \chi_{E} \in L^{q_{1}}(\Omega)$ and $u_{n} \chi_{E^{c}} \in L^{q_{2}}(\Omega)$ and thus, by definition (2.2), one has $\left\|u_{n}\right\| \leq\left\|u_{n}\right\|_{L^{q_{1}(E)}}+\left\|u_{n}\right\|_{L^{q_{2}\left(E^{c}\right)}}<\varepsilon^{1 / q_{1}}+\varepsilon^{1 / q_{2}}$ for all $n>n_{\varepsilon}$, with $\varepsilon$ arbitrary.

Proposition 2.8. The convergence in $L(\Omega)$ implies pointwise convergence ( $\mu$-a.e. and up to a subsequence).

Proof. Let $\left\{u_{n}\right\} \subseteq L(\Omega)$ be such that $\left\|u_{n}\right\|_{1} \rightarrow 0$ as $n \rightarrow \infty$. Then for every $n>0$ there exists $\left(u_{1, n}, u_{2, n}\right) \in L^{q_{1}}(\Omega) \times L^{q_{2}}(\Omega)$ such that $u_{n}=u_{1, n}+u_{2, n}$ and $\left\|\left(u_{1, n}, u_{2, n}\right)\right\|_{1} \leq\left\|u_{n}\right\|_{1}+1 / n$. Hence $u_{1, n} \rightarrow 0$ in $L^{q_{1}}(\Omega)$ and $u_{2, n} \rightarrow 0$ in $L^{q_{2}}(\Omega)$, which implies that, up to a subsequence, $u_{1, n}, u_{2, n} \rightarrow 0 \mu$-a.e. in $\Omega$. Therefore $u_{n}=u_{1, n}+u_{2, n} \rightarrow 0 \mu$-a.e. in $\Omega$.

We now prove the most important result of this section, an isometrical identification between $\left(L(\Omega),\|\cdot\|^{*}\right)$ and the dual space of $\left(L^{q_{1}^{\prime}}(\Omega) \cap L^{q_{2}^{\prime}}(\Omega)\right.$, $\|\cdot\|_{L^{q_{1}^{\prime} \cap L^{q_{2}^{\prime}}}}$, where

$$
q_{i}^{\prime}=\frac{q_{i}}{q_{i}-1} \quad \text { and } \quad\|\varphi\|_{L^{q_{1}^{\prime} \cap L^{q_{2}^{\prime}}}}:=\|\varphi\|_{L^{q_{1}^{\prime}(\Omega)}}+\|\varphi\|_{L^{q_{2}^{\prime}(\Omega)}} .
$$

This will also ensure that $L(\Omega)$ is a reflexive Banach space (see Corollary 2.11 below). For future reference, let us give first the following lemma.

Lemma 2.9. For any $u \in L(\Omega)$ and $\varphi \in L^{q_{1}^{\prime}}(\Omega) \cap L^{q_{2}^{\prime}}(\Omega)$ one has

$$
\int_{\Omega}|u \varphi| d \mu \leq\|u\|^{*}\|\varphi\|_{L^{q_{1}^{\prime} \cap L^{q_{2}^{\prime}}}}
$$

and

$$
\int_{\Omega}|u \varphi| d \mu \leq\|u\| \max \left\{\|\varphi\|_{L^{q_{1}^{\prime}(\Omega)}},\|\varphi\|_{L^{q_{2}^{\prime}(\Omega)}}\right\} .
$$


Proof. Let $u \in L(\Omega)$ and let $u_{1} \in L^{q_{1}}(\Omega)$ and $u_{2} \in L^{q_{2}}(\Omega)$ be such that $u_{1}+u_{2}=u$. Then, by Hölder inequality, $\forall \varphi \in L^{q_{1}^{\prime}}(\Omega) \cap L^{q_{2}^{\prime}}(\Omega)$ one has

$$
\begin{aligned}
\int_{\Omega}|u \varphi| d \mu & \leq \int_{\Omega}\left|u_{1} \varphi\right| d \mu+\int_{\Omega}\left|u_{2} \varphi\right| d \mu \\
& \leq\left\|u_{1}\right\|_{L^{q_{1}(\Omega)}}\|\varphi\|_{L^{q_{1}^{\prime}(\Omega)}}+\left\|u_{2}\right\|_{L^{q_{2}(\Omega)}}\|\varphi\|_{L^{q_{2}^{\prime}(\Omega)}},
\end{aligned}
$$

which easily yields the result.

Theorem 2.10. For any $u \in L(\Omega)$ and $\varphi \in L^{q_{1}^{\prime}}(\Omega) \cap L^{q_{2}^{\prime}}(\Omega)$,

$$
J(u) \varphi:=\int_{\Omega} u \varphi d \mu
$$

defines a linear continuous functional $J(u): L^{q_{1}^{\prime}}(\Omega) \cap L^{q_{2}^{\prime}}(\Omega) \rightarrow \mathbb{R}$. Moreover the linear operator $J: L(\Omega) \rightarrow\left(L^{q_{1}^{\prime}}(\Omega) \cap L^{q_{2}^{\prime}}(\Omega)\right)^{\prime}$ is bijective and one has

$$
\|u\|^{*}=\sup _{0 \neq \varphi \in L^{q_{1}^{\prime}}(\Omega) \cap L^{q_{2}^{\prime}(\Omega)}} \frac{\int_{\Omega} u \varphi d \mu}{\|\varphi\|_{L_{1}^{q_{1}^{\prime}(\Omega)}}+\|\varphi\|_{L^{q_{2}^{\prime}}(\Omega)}} \quad \text { for every } u \in L(\Omega) .
$$

Proof. Denote $L:=L(\Omega)$ and $L^{p}:=L^{p}(\Omega)$ for brevity.

We begin with some preliminary remarks about the dual space $\left(L^{q_{1}^{\prime}} \cap L^{q_{2}^{\prime}}\right)^{\prime}$ of $L^{q_{1}^{\prime}} \cap L^{q_{2}^{\prime}}$ equipped with the norm (2.8). First we observe that there is a natural linear isometry between $L^{q_{1}^{\prime}} \cap L^{q_{2}^{\prime}}$ and the closed subspace

$$
\Delta:=\left\{(\varphi, \psi) \in L^{q_{1}^{\prime}} \times L^{q_{2}^{\prime}}: \varphi=\psi\right\}
$$

of the Banach space $L^{q_{1}^{\prime}} \times L^{q_{2}^{\prime}}$ equipped with the norm $\|(\varphi, \psi)\|_{L^{q_{1}^{\prime}} \times L^{q_{2}^{\prime}}}:=$ $\|\varphi\|_{L^{q_{1}^{\prime}}}+\|\psi\|_{L^{q_{2}^{\prime}}}$. Hence $\left(L^{q_{1}^{\prime}} \cap L^{q_{2}^{\prime}}\right)^{\prime}$ isometrically identifies with the dual space $\Delta^{\prime}$, so that for any $g \in\left(L^{q_{1}^{\prime}} \cap L^{q_{2}^{\prime}}\right)^{\prime}$ there exists a unique $G \in \Delta^{\prime}$ such that $\|G\|_{\Delta^{\prime}}=\|g\|_{\left(L^{q_{1}^{\prime}} \cap L^{q_{2}^{\prime}}\right)^{\prime}}$ and $G(\varphi, \varphi)=g(\varphi)$ for all $\varphi \in L^{q_{1}^{\prime}} \cap L^{q_{2}^{\prime}}$. Then, by the Hahn-Banach theorem, there exists $\widetilde{G} \in\left(L^{q_{1}^{\prime}} \times L^{q_{2}^{\prime}}\right)^{\prime}$ such that $\|\widetilde{G}\|_{\left(L^{q_{1}^{\prime}} \times L^{q_{2}^{\prime}}\right)^{\prime}}=\|g\|_{\left(L^{\left.q_{1}^{\prime} \cap L_{2}^{q_{2}^{\prime}}\right)^{\prime}}\right.}$ and $\widetilde{G}(\varphi, \varphi)=g(\varphi)$ for all $\varphi \in L^{q_{1}^{\prime}} \cap L^{q_{2}^{\prime}}$. Now, since $\widetilde{G}(\varphi, \psi)=\widetilde{G}(\varphi, 0)+\widetilde{G}(0, \psi)$ with $\widetilde{G}(\cdot, 0) \in\left(L^{q_{1}^{\prime}}\right)^{\prime}$ and $\widetilde{G}(0, \cdot) \in\left(L^{q_{2}^{\prime}}\right)^{\prime}$, by the Riesz representation theorem there exist $v_{1} \in L^{q_{1}}$ and $v_{2} \in L^{q_{2}}$ such that

$$
\forall(\varphi, \psi) \in L^{q_{1}^{\prime}} \times L^{q_{2}^{\prime}}, \quad \widetilde{G}(\varphi, \psi)=\int_{\Omega} v_{1} \varphi d \mu+\int_{\Omega} v_{2} \psi d \mu .
$$

This gives in particular

$$
\forall \varphi \in L^{q_{1}^{\prime}} \cap L^{q_{2}^{\prime}} \quad g(\varphi)=\int_{\Omega}\left(v_{1}+v_{2}\right) \varphi d \mu .
$$

Moreover if $v_{1} \neq 0$ then

$$
\|\widetilde{G}\|_{\left(L^{q_{1}^{\prime} \times L^{\left.q_{2}^{\prime}\right)^{\prime}}}\right.}=\sup _{(\varphi, \psi) \neq(0,0)} \frac{\int_{\Omega} v_{1} \varphi d \mu+\int_{\Omega} v_{2} \psi d \mu}{\|\varphi\|_{L^{q_{1}^{\prime}}}+\|\psi\|_{L^{q_{2}^{\prime}}}} \geq \frac{\int_{\Omega}\left|v_{1}\right|^{q_{1}} d \mu}{\left\|v_{1}\right\|_{L^{q_{1}}}^{q_{1} / q_{1}^{\prime}}}=\left\|v_{1}\right\|_{L^{q_{1}}}
$$


where, for the inequality, we have taken $\psi=0$ and $\varphi=\left|v_{1}\right|^{q_{1}-2} v_{1} \in L^{q_{1}^{\prime}}$. Similarly one obtains $\|\widetilde{G}\|_{\left(L^{q_{1}^{\prime}} \times L^{q_{2}^{\prime}}\right)^{\prime}} \geq\left\|v_{2}\right\|_{L^{q_{2}}}$ if $v_{2} \neq 0$. Thus we conclude

$$
\|g\|_{\left(L^{\left.q_{1}^{\prime} \cap L^{q_{2}^{\prime}}\right)^{\prime}}\right.}=\|\widetilde{G}\|_{\left(L^{q_{1}^{\prime}} \times L^{q_{2}^{\prime}}\right)^{\prime}} \geq \max \left\{\left\|v_{1}\right\|_{L^{q_{1}}},\left\|v_{2}\right\|_{L^{q_{2}}}\right\} \geq\left\|v_{1}+v_{2}\right\|^{*},
$$

which trivially holds even when $v_{1}=v_{2}=0$.

Now let $u \in L$ and let $u_{1} \in L^{q_{1}}$ and $u_{2} \in L^{q_{2}}$ be such that $u_{1}+u_{2}=u$. For every $\varphi \in L^{q_{1}^{\prime}} \cap L^{q_{2}^{\prime}}$ one has

$$
\begin{aligned}
& \left|\int_{\Omega} u \varphi d \mu\right| \leq \int_{\Omega}\left|u_{1} \varphi d \mu\right|+\int_{\Omega}\left|u_{2} \varphi d \mu\right| \leq\left\|u_{1}\right\|_{L^{q_{1}}}\|\varphi\|_{L^{q_{1}^{\prime}}}+\left\|u_{2}\right\|_{L^{q_{2}}}\|\varphi\|_{L^{q_{2}^{\prime}}} \\
& \leq \max \left\{\left\|u_{1}\right\|_{L^{q_{1}}},\left\|u_{2}\right\|_{L^{q_{2}}}\right\}\|\varphi\|_{L^{q_{1}^{\prime} \cap L^{q_{2}^{\prime}}}} .
\end{aligned}
$$

Hence $J(u) \in\left(L^{q_{1}^{\prime}} \times L^{q_{2}^{\prime}}\right)^{\prime}$ and

$$
\|J(u)\|_{\left(L^{\left.q_{1}^{\prime} \cap L^{q_{2}^{\prime}}\right)^{\prime}} \leq\right.} \leq\|u\|^{*},
$$

which also implies that the linear operator $J: L \rightarrow\left(L^{q_{1}^{\prime}} \cap L^{q_{2}^{\prime}}\right)^{\prime}$ is continuous. The injectivity of $J$ is plain, as $\int_{\Omega} u \varphi d \mu=0$ for all $\varphi \in L^{q_{1}^{\prime}} \cap L^{q_{2}^{\prime}}$ implies $u=0$ $\mu$-a.e. in $\Omega$. Moreover $J$ is surjective by the previous preliminary discussion, since (2.10) ensures that for any $g \in\left(L^{q_{1}^{\prime}} \cap L^{q_{2}^{\prime}}\right)^{\prime}$ there exist $v_{1} \in L^{q_{1}}$ and $v_{2} \in L^{q_{2}}$ such that $g=J\left(v_{1}+v_{2}\right)$. Finally, if $u \in L$ is fixed, then (2.12) and (2.11) (in which we take $g=J(u)$ ) yield

$$
\|u\|^{*} \geq\|J(u)\|_{\left(L^{\left.q_{1}^{\prime} \cap L^{q_{2}^{\prime}}\right)^{\prime}}\right.} \geq\left\|v_{1}+v_{2}\right\|^{*}=\|u\|^{*}
$$

where the last equality holds thanks to the injectivity of $J$, because $(2.10)$ means $J(u)=J\left(v_{1}+v_{2}\right)$.

Corollary 2.11. $L(\Omega)$ is a reflexive Banach space.

Proof. It follows from the completeness and reflexivity of $L^{q_{1}^{\prime}}(\Omega) \cap L^{q_{2}^{\prime}}(\Omega)$, by Theorem 2.10.

Corollary 2.12. Let $u \in \mathcal{M}(\Omega)$ and $v \in L(\Omega)$. Then $|u| \leq v$ implies $\|u\|^{*} \leq$ $\|v\|^{*}$.

Proof. Recall that $|u| \leq v \in L(\Omega)$ implies $|u| \in L(\Omega)$ by Proposition 2.3. Since $0 \leq|u| \leq v$, one has

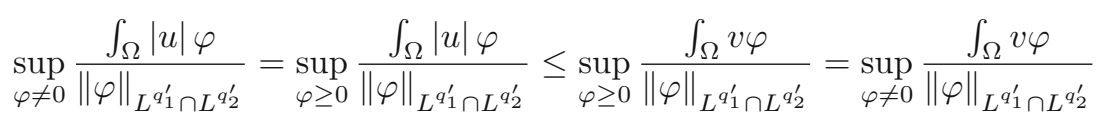

where $\varphi$ varies in $L^{q_{1}^{\prime}}(\Omega) \cap L^{q_{2}^{\prime}}(\Omega)$. Then $\|u\|^{*}=\||u|\|^{*} \leq\|v\|^{*}$ by Proposition 2.4 and Theorem 2.10 .

\subsection{Some inequalities and continuous embeddings}

Recall from Proposition 2.1 that $L(\Omega) \cap L^{\infty}(E) \subseteq L^{q_{2}}(E)$ for any measurable $E \subseteq \Omega$, and $L(\Omega) \subseteq L^{q_{1}}(E)$ if $\mu(E)<+\infty$. 
Proposition 2.13. Let $u \in L(\Omega)$ and let $E \subseteq \Omega$ be a measurable set such that $\mu(E)<+\infty$ and $u \in L^{\infty}\left(E^{c}\right)$. Then

$$
\begin{gathered}
\|u\|^{*} \leq \max \left\{\|u\|_{L^{q_{1}}(E)},\|u\|_{L^{q_{2}}\left(E^{c}\right)}\right\}, \\
\|u\| \leq\|u\|_{L^{q_{1}}(E)}+\|u\|_{L^{q_{2}}\left(E^{c}\right)} .
\end{gathered}
$$

Proof. From Proposition 2.1 we know that $u \chi_{E} \in L^{q_{1}}(\Omega)$ and $u \chi_{E^{c}} \in L^{q_{2}}(\Omega)$. Hence the claim follows by definitions (2.2) and (2.4), since $u=u \chi_{E}+u \chi_{E^{c}}$.

Proposition 2.1 can be complemented by the following result.

Proposition 2.14. Let $E \subseteq \Omega$ be a measurable set.

(i) If $\mu(E)<+\infty$, then for every $u \in L(\Omega)$ one has

$$
\begin{gathered}
\|u\|_{L^{q_{1}}(E)} \leq\left(1+\mu(E)^{1 / q_{1}-1 / q_{2}}\right)\|u\|^{*}, \\
\|u\|_{L^{q_{1}}(E)} \leq \max \left\{1, \mu(E)^{1 / q_{1}-1 / q_{2}}\right\}\|u\| .
\end{gathered}
$$

(ii) For every $u \in L(\Omega) \cap L^{\infty}(E)$ one has

$$
\begin{aligned}
& \|u\|_{L^{q_{2}(E)}}^{q_{2} / q_{1}} \leq\left(\|u\|_{L^{\infty}(E)}^{q_{2} / q_{1}-1}+\|u\|_{L^{q_{2}(E)}}^{q_{2} / q_{1}-1}\right)\|u\|^{*}, \\
& \|u\|_{L^{q_{2}(E)}}^{q_{2} / q_{1}} \leq \max \left\{\|u\|_{L^{\infty}(E)}^{q_{2} / q_{1}-1},\|u\|_{L^{q_{2}}(E)}^{q_{2} / q_{1}-1}\right\}\|u\| .
\end{aligned}
$$

Proof. First we prove (2.17)-(2.18), which are is obvious if $u=0$. Accordingly, assume $u \in L(\Omega) \cap L^{\infty}(E), u \neq 0$, and define

$$
\varphi_{u}:=\frac{|u|^{q_{2}-2} u}{\|u\|_{L^{q_{2}(E)}}^{q_{2}-1}} \chi_{E} .
$$

Then

$$
\int_{\Omega}\left|\varphi_{u}\right|^{q_{2}^{\prime}} d \mu=\frac{1}{\|u\|_{L^{q_{2}(E)}}^{q_{2}}} \int_{E}|u|^{q_{2}} d \mu=1
$$

and

$$
\begin{aligned}
\int_{\Omega}\left|\varphi_{u}\right|^{q_{1}^{\prime}} d \mu & =\frac{1}{\|u\|_{L^{q_{2}(E)}}^{\left(q_{2}-1\right) q_{1}^{\prime}}} \int_{E}|u|^{\left(q_{2}-1\right) q_{1}^{\prime}} d \mu \\
& \leq \frac{\|u\|_{L^{\infty}(E)}^{\left(q_{2}-1\right) q_{1}^{\prime}-q_{2}}}{\|u\|_{L^{q_{2}}(E)}^{\left(q_{2}-1\right) q_{1}^{\prime}}} \int_{E}|u|^{q_{2}} d \mu=\frac{\|u\|_{L^{\infty}(E)}^{\left(q_{2}-1\right) q_{1}^{\prime}-q_{2}}}{\|u\|_{L^{q_{2}}(E)}^{\left(q_{2}-1\right) q_{1}^{\prime}-q_{2}}}
\end{aligned}
$$

since $q_{1}<q_{2}$ implies $\left(q_{2}-1\right) q_{1}^{\prime}>q_{2}$. So $\varphi_{u} \in L^{q_{1}^{\prime}}(\Omega) \cap L^{q_{2}^{\prime}}(\Omega)$ and thus, since

$$
\int_{\Omega} u \varphi_{u} d \mu=\frac{1}{\|u\|_{L^{q_{2}(E)}}^{q_{2}-1}} \int_{E}|u|^{q_{2}} d \mu=\|u\|_{L^{q_{2}(E)}}
$$


Lemma 2.9 gives

$$
\|u\|_{L^{q_{2}(E)}} \leq\|u\|^{*}\left(\left\|\varphi_{u}\right\|_{L^{q_{1}^{\prime}(\Omega)}}+\left\|\varphi_{u}\right\|_{L^{q_{2}^{\prime}(\Omega)}}\right) \leq\|u\|^{*}\left(\frac{\|u\|_{L^{\infty}(E)}^{q_{2}-1-q_{2} / q_{1}^{\prime}}}{\|u\|_{L^{q_{2}(E)}}^{q_{2}-1-q_{2} / q_{1}^{\prime}}}+1\right)
$$

and

$$
\begin{aligned}
\|u\|_{L^{q_{2}}(E)} & \leq\|u\| \max \left\{\left\|\varphi_{u}\right\|_{L^{q_{1}^{\prime}}(\Omega)},\left\|\varphi_{u}\right\|_{L^{q_{2}^{\prime}(\Omega)}}\right\} \\
& \leq\|u\| \max \left\{\frac{\|u\|_{L^{\infty}(E)}^{q_{2}-1-q_{2} / q_{1}^{\prime}}}{\|u\|_{L^{q_{2}}(E)}^{q_{2}-1-q_{2} / q_{1}^{\prime}}}, 1\right\},
\end{aligned}
$$

which yield the results, since $q_{2}-1-q_{2} / q_{1}^{\prime}=q_{2} / q_{1}-1$ and

$$
\max \left\{\frac{\|u\|_{L^{\infty}(E)}^{q_{2} / q_{1}-1}}{\|u\|_{L^{q_{2}(E)}}^{q_{2} / q_{1}-1}}, 1\right\}=\frac{\max \left\{\|u\|_{L^{\infty}(E)}^{q_{2} / q_{1}-1},\|u\|_{L^{q_{2}}(E)}^{q_{2} / q_{1}-1}\right\}}{\|u\|_{L^{q_{2}(E)}}^{q_{2} / q_{1}-1}} .
$$

Now we let $u \in L(\Omega)$, assume that $\mu(E)<+\infty$ and let $u_{1} \in L^{q_{1}}(\Omega)$ and $u_{2} \in L^{q_{2}}(\Omega)$ be such that $u=u_{1}+u_{2}$. By Hölder inequality we get

$$
\begin{aligned}
\|u\|_{L^{q_{1}}(E)} & \leq\left\|u_{1}\right\|_{L^{q_{1}}(E)}+\left\|u_{2}\right\|_{L^{q_{1}}(E)} \\
& \leq\left\|u_{1}\right\|_{L^{q_{1}}(E)}+\mu(E)^{1 / q_{1}-1 / q_{2}}\left\|u_{2}\right\|_{L^{q_{2}}(E)},
\end{aligned}
$$

whence

$$
\begin{aligned}
& \|u\|_{L^{q_{1}}(E)} \leq\left(1+\mu(E)^{1 / q_{1}-1 / q_{2}}\right) \max \left\{\left\|u_{1}\right\|_{L^{q_{1}}(E)},\left\|u_{2}\right\|_{L^{q_{2}}(E)}\right\} \\
& \|u\|_{L^{q_{1}}(E)} \leq \max \left\{1, \mu(E)^{1 / q_{1}-1 / q_{2}}\right\}\left(\left\|u_{1}\right\|_{L^{q_{1}}(E)}+\left\|u_{2}\right\|_{L^{q_{2}}(E)}\right) .
\end{aligned}
$$

Then (2.15)-(2.16) ensue by passing to the infima.

Recall that $L(\Omega)$ and $L^{q_{1}}(\Omega)$ are the same set if $\mu(\Omega)<+\infty$.

Corollary 2.15. If $\mu(\Omega)<+\infty$, the norms of $L(\Omega)$ and $L^{q_{1}}(\Omega)$ are equivalent.

Proof. One has $\|u\|^{*} \leq\|u\|_{L^{q_{1}}(\Omega)} \leq\left(1+\mu(\Omega)^{1 / q_{1}-1 / q_{2}}\right)\|u\|^{*}$ by (2.13) and (2.15).

Recall from Corollary 2.2 that $L(\Omega) \cap L^{\infty}(\Omega)$ and $L^{q_{2}}(\Omega) \cap L^{\infty}(\Omega)$ are the same set.

Corollary 2.16. On the subspace $L^{q_{2}}(\Omega) \cap L^{\infty}(\Omega)$ of $L(\Omega)$, the norms $\|u\|+$ $\|u\|_{L^{\infty}(\Omega)}$ and $\|u\|_{L^{q_{2}(\Omega)}}+\|u\|_{L^{\infty}(\Omega)}$ are equivalent.

Proof. If $u \in L^{q_{2}}(\Omega) \cap L^{\infty}(\Omega)$ then (2.14), with $E=\varnothing$, gives $\|u\| \leq\|u\|_{L^{q_{2}}(\Omega)}$. On the other hand, if $\left\{u_{n}\right\} \subseteq L^{q_{2}}(\Omega) \cap L^{\infty}(\Omega)$ is such that $\left\|u_{n}\right\|+\left\|u_{n}\right\|_{L^{\infty}(\Omega)} \rightarrow$ 0 , then (2.18) yields

$$
\left\|u_{n}\right\|_{L^{q_{2}}(\Omega)}^{q_{2} / q_{1}} \leq\left(1+\left\|u_{n}\right\|_{L^{q_{2}(\Omega)}}^{q_{2} / q_{1}-1}\right) o(1) \quad \text { as } n \rightarrow \infty
$$

which implies $\left\|u_{n}\right\|_{L^{q_{2}(\Omega)}} \rightarrow 0$. 
The next proposition collects some embedding properties of $L(\Omega)$, some of which are consequences of the above inequalities. Another relevant embedding result will be proved in Sect. 4 (Theorem 4.1) for $\Omega=\mathbb{R}^{N}$.

Proposition 2.17. The following continuous embeddings hold:

(i) $L(\Omega) \hookrightarrow L(E)$ for any measurable $E \subseteq \Omega$, namely, for every $u \in L(\Omega)$ one has $\chi_{E} u \in L(E)$ and $\left\|\chi_{E} u\right\|_{L(E)} \leq\|u\|_{L(\Omega)}$;

(ii) $L(\Omega) \hookrightarrow L^{q_{1}}(E)$ for any measurable $E \subseteq \Omega$ such that $\mu(E)<+\infty$;

(iii) $L^{q}(\Omega) \hookrightarrow L(\Omega)$ for any $q \in\left[q_{1}, q_{2}\right]$.

Proof. The first and second continuous embeddings straightforwardly follow from definition (2.2) and Proposition 2.14.i respectively. So we take $q \in\left[q_{1}, q_{2}\right]$ and show that $L^{q}(\Omega) \hookrightarrow L(\Omega)$. Let $u \in L^{q}(\Omega)$ and recall the definition (2.1) of $\Lambda_{u}$. Then $q_{1} \leq q$ and

$$
\mu\left(\Lambda_{u}\right)=\int_{\Lambda_{u}} d \mu \leq \int_{\Lambda_{u}}|u|^{q} d \mu \leq\|u\|_{L^{q}(\Omega)}^{q}<+\infty
$$

imply $u \in L^{q_{1}}\left(\Lambda_{u}\right)$ and, by Hölder inequality,

$$
\begin{aligned}
\int_{\Lambda_{u}}|u|^{q_{1}} d \mu & \leq \mu\left(\Lambda_{u}\right)^{1-q_{1} / q}\left(\int_{\Lambda_{u}}|u|^{q} d \mu\right)^{q_{1} / q} \leq\|u\|_{L^{q}(\Omega)}^{q\left(1-q_{1} / q\right)}\|u\|_{L^{q}(\Omega)}^{q_{1}} \\
& =\|u\|_{L^{q}(\Omega)}^{q} .
\end{aligned}
$$

On the other hand, $q \leq q_{2}$ and $|u| \leq 1$ imply $|u|^{q_{2}} \leq|u|^{q}$, so that $u \in L^{q_{2}}\left(\Lambda_{u}^{c}\right)$ and

$$
\int_{\Lambda_{u}^{c}}|u|^{q_{2}} d \mu \leq \int_{\Lambda_{u}^{c}}|u|^{q} d \mu \leq\|u\|_{L^{q}(\Omega)}^{q} .
$$

Thus $u \in L(\Omega)$ since $u=u \chi_{\Lambda_{u}}+u \chi_{\Lambda_{u}^{c}}$, and Proposition 2.13 gives

$$
\|u\| \leq\|u\|_{L^{q_{1}\left(\Lambda_{u}\right)}}+\|u\|_{L^{q_{2}\left(\Lambda_{u}^{c}\right)}} \leq\|u\|_{L^{r}(\Omega)}^{q / q_{1}}+\|u\|_{L^{r}(\Omega)}^{q / q_{2}} .
$$

This implies that $\left\|u_{n}\right\| \rightarrow 0$ if $\left\|u_{n}\right\|_{L^{q}(\Omega)} \rightarrow 0$ and the proof is thus complete.

Remark 2.18. On $L^{q}(\Omega), q_{1} \leq q \leq q_{2}$, the norms (2.2)-(2.4) and the standard $L^{q}(\Omega)$ norm are not equivalent in general. For this, we have two different counterexamples, according as $q_{1} \leq q<q_{2}$ or $q=q_{2}$, both for $\Omega=\mathbb{R}^{N}$ endowed with the Lebesgue measure. In the first case, $q \in\left[q_{1}, q_{2}\right)$, define the sequence

$$
u_{n}(x):= \begin{cases}1 / n & \text { if }|x| \leq n^{q_{2} / N} \\ 0 & \text { otherwise }\end{cases}
$$

Then one has

$$
\left\|u_{n}\right\|_{L^{q}\left(\mathbb{R}^{N}\right)}^{q}=\frac{1}{n^{q}} \int_{|x| \leq n^{q_{2} / N}} d x=\text { (const.) } n^{q_{2}-q} \rightarrow+\infty,
$$

whereas (2.14) (with $E=\varnothing)$ gives

$$
\left.\left\|u_{n}\right\| \leq\left(\int_{|x| \leq n^{q_{2} / N}} \frac{1}{n^{q_{2}}} d x\right)^{1 / q_{2}}=\frac{1}{n}\left(\int_{|x| \leq n^{q_{2} / N}} d x\right)^{1 / q_{2}}=\text { (const. }\right) .
$$


Similarly, if $q=q_{2}$, the sequence

$$
u_{n}(x):= \begin{cases}n & \text { if }|x| \leq n^{-q_{1} / N} \\ 0 & \text { otherwise }\end{cases}
$$

is bounded in $L(\Omega)$ (by (2.14) again, with $E=B_{n^{-q_{1} / N}}$ ) and such that $\left\|u_{n}\right\|_{L^{q_{2}\left(\mathbb{R}^{N}\right)}}^{q_{2}} \rightarrow+\infty$.

The next result is a corollary of Propositions 2.13 and 2.14.

Corollary 2.19. Let $u \in L(\Omega)$ and recall the definition (2.1) of $\Lambda_{u}$. One has

$$
\begin{aligned}
& \max \left\{\frac{\|u\|_{L^{q_{1}\left(\Lambda_{u}\right)}}}{1+\mu\left(\Lambda_{u}\right)^{1 / q_{1}-1 / q_{2}}}, \frac{1}{2}\|u\|_{L^{q_{2}\left(\Lambda_{u}^{c}\right)}}-\frac{1}{2}\right\} \leq\|u\|^{*} \\
& \quad \leq \max \left\{\|u\|_{L^{q_{1}\left(\Lambda_{u}\right)}},\|u\|_{L^{q_{2}\left(\Lambda_{u}^{c}\right)}}\right\}
\end{aligned}
$$

and

$$
\begin{aligned}
& \max \left\{\frac{\|u\|_{L^{q_{1}}\left(\Lambda_{u}\right)}}{\max \left\{1, \mu\left(\Lambda_{u}\right)^{1 / q_{1}-1 / q_{2}}\right\}},\|u\|_{L^{q_{2}\left(\Lambda_{u}^{c}\right)}}-l\right\} \leq\|u\| \\
& \leq\|u\|_{L^{q_{1}\left(\Lambda_{u}\right)}+\|u\|_{L^{q_{2}}\left(\Lambda_{u}^{c}\right)}},
\end{aligned}
$$

where $l:=\left(q_{2}-q_{1}\right)\left(q_{1} / q_{2}\right)^{q_{2} /\left(q_{2}-q_{1}\right)} / q_{1}$.

Proof. Since $\|u\|_{L^{\infty}\left(\Lambda_{u}^{c}\right)} \leq 1$ and $\mu\left(\Lambda_{u}\right)<+\infty$ (recall Proposition 2.3), the right hand inequalities of (2.20)-(2.21) and part of the left hand ones directly follow from Propositions 2.13 and 2.14 respectively. Then, setting $t:=$ $\|u\|_{L^{q_{2}\left(\Lambda_{u}^{c}\right)}}$, from (2.18) we get

$$
\frac{t^{q_{2} / q_{1}}}{\max \left\{1, t^{q_{2} / q_{1}-1}\right\}} \leq\|u\|,
$$

so that the remaining part of (2.21) follows from the inequality

$$
\frac{t^{q_{2} / q_{1}}}{\max \left\{1, t^{q_{2} / q_{1}-1}\right\}}-t \geq-l
$$

which holds for every $t \geq 0$. Finally we show that

$$
\|u\|_{L^{q_{2}\left(\Lambda_{u}^{c}\right)}} \leq \max \left(2\|u\|^{*}, 1\right),
$$

which completes the proof of (2.20). To this end, we set $t:=\|u\|_{L^{q_{2}\left(\Lambda_{u}^{c}\right)}}^{q_{2} / q_{1}-1}$ and use (2.17) to deduce

$$
\frac{t}{1+t}\|u\|_{L^{q_{2}\left(\Lambda_{u}^{c}\right)}} \leq\|u\|^{*}
$$

Then $\|u\|_{L^{q_{2}\left(\Lambda_{u}^{c}\right)}}>1$ implies $t>1$ (recall that $q_{2} / q_{1}>1$ ) and thus we get $\|u\|_{L^{q_{2}\left(\Lambda_{u}^{c}\right)}} \leq 2\|u\|^{*}$, since $t>(1+t) / 2$.

Remark 2.20. Note that, according to the above proof of Corollary 2.19, the inequality $\|u\|_{L^{q_{2}\left(\Lambda_{u}^{c}\right)}}-1 \leq 2\|u\|^{*}$ of (2.20) actually holds in the stronger form

$$
\|u\|_{L^{q_{2}\left(\Lambda_{u}^{c}\right)}} \leq \max \left(2\|u\|^{*}, 1\right) .
$$


On the other hand, inequalities (2.20)-(2.21) cannot be improved in the following sense: there is no constant $C>0$ such that

$$
\|u\|_{L^{q_{2}\left(\Lambda_{u}^{c}\right)}} \leq C\|u\|^{*} \quad \text { for all } u \in L(\Omega) .
$$

Indeed, arguing by contradiction, for any $u \in L(\Omega) \cap L^{\infty}(\Omega), u \neq 0$, we set $\tilde{u}:=u /\|u\|_{L^{\infty}(\Omega)}\left(\right.$ so that $\Lambda_{\tilde{u}}=\varnothing$ ) and by (2.22) we obtain

$$
C \frac{\|u\|^{*}}{\|u\|_{L^{\infty}(\Omega)}}=C\|\tilde{u}\|^{*} \geq\|\tilde{u}\|_{L^{q_{2}\left(\Lambda_{u}^{c}\right)}}=\|\tilde{u}\|_{L^{q_{2}(\Omega)}}=\frac{\|u\|_{L^{q_{2}}(\Omega)}}{\|u\|_{L^{\infty}(\Omega)}},
$$

that is,

$$
\|u\|_{L^{q_{2}(\Omega)}} \leq C\|u\|^{*} .
$$

But the sequence $\left\{u_{n}\right\} \subseteq L(\Omega) \cap L^{\infty}(\Omega)$ defined in (2.19) does not satisfy (2.23) and thus a contradiction ensues.

We end this section with a characterization of the boundedness of a sequence in $L(\Omega)$.

Proposition 2.21. Let $\left\{u_{n}\right\}$ be a sequence in $L(\Omega)$ and denote $\Lambda_{n}:=\Lambda_{u_{n}}$. Then $\left\{u_{n}\right\}$ is bounded in $L(\Omega)$ if and only if $\left\{\left\|u_{n}\right\|_{L^{q_{1}}\left(\Lambda_{n}\right)}\right\},\left\{\left\|u_{n}\right\|_{L^{q_{2}\left(\Lambda_{n}^{c}\right)}}\right\}$ and $\left\{\mu\left(\Lambda_{n}\right)\right\}$ are bounded.

Proof. The "if part" directly follows from Corollary 2.19, which readily gives also that $\left\{\left\|u_{n}\right\|_{L^{q_{2}\left(\Lambda_{n}^{c}\right)}}\right\}$ is bounded if $\left\{u_{n}\right\}$ is bounded in $L(\Omega)$. We now use an argument from [15]: since $\left|u_{n}\right|>1$ on $\Lambda_{n}$, from (2.20) we get $\left\|u_{n}\right\|^{*} \geq \frac{\left\|u_{n}\right\|_{L^{q_{1}}\left(\Lambda_{u}\right)}}{1+\mu\left(\Lambda_{u}\right)^{1 / q_{1}-1 / q_{2}}} \geq \frac{\mu\left(\Lambda_{n}\right)^{1 / q_{1}}}{1+\mu\left(\Lambda_{n}\right)^{1 / q_{1}-1 / q_{2}}} \rightarrow \infty \quad$ as $\mu\left(\Lambda_{n}\right) \rightarrow \infty$

and therefore the boundedness of $\left\{\left\|u_{n}\right\|^{*}\right\}$ implies the one of $\left\{\mu\left(\Lambda_{n}\right)\right\}$, and then of $\left\{\left\|u_{n}\right\|_{L^{q_{1}\left(\Lambda_{n}\right)}}\right\}$.

\subsection{The Orlicz structure of $L(\Omega)$}

Define

$$
\phi(t):=\min \left\{t^{q_{1}-1}, t^{q_{2}-1}\right\} \quad \text { for all } t \geq 0
$$

and set

$$
\Phi(t):=\int_{0}^{|t|} \phi(s) d s \quad \text { for all } t \in \mathbb{R}
$$

that is,

$$
\Phi(t)= \begin{cases}\frac{1}{q_{2}}|t|^{q_{2}} & \text { if }|t| \leq 1 \\ \frac{1}{q_{1}}|t|^{q_{1}}+\frac{1}{q_{2}}-\frac{1}{q_{1}} & \text { if }|t|>1\end{cases}
$$

Then $\Phi: \mathbb{R} \rightarrow[0,+\infty)$ is a nice Young function, i.e., an even, convex and continuous function such that

$$
\lim _{t \rightarrow 0} \frac{\Phi(t)}{t}=0, \quad \lim _{t \rightarrow+\infty} \frac{\Phi(t)}{t}=+\infty \quad \text { and } \quad \Phi(t)=0 \Leftrightarrow t=0,
$$


and we can consider the Orlicz class

$$
L^{\Phi}(\Omega):=\left\{u \in \mathcal{M}(\Omega): \int_{\Omega} \Phi(u) d \mu<+\infty\right\} .
$$

We will show that $L^{\Phi}(\Omega)$ is exactly $L(\Omega)$. Note that $\Phi$ satisfies the so-called global $\Delta_{2}$ condition, that is, there exists $\eta>0$ such that

$$
\Phi(2 t) \leq \eta \Phi(t) \quad \text { for all } t \geq 0,
$$

so that $L^{\Phi}(\Omega)$ is a vector space (see [34, Theorem 3.2]).

Proposition 2.22. One has $L^{\Phi}(\Omega)=L(\Omega)$.

Proof. By (2.24), for any $u \in \mathcal{M}(\Omega)$ we have

$$
\int_{\Omega} \Phi(u) d \mu=\frac{1}{q_{1}} \int_{\Lambda_{u}}\left(|t|^{q_{1}}-1\right) d \mu+\frac{1}{q_{2}} \mu\left(\Lambda_{u}\right)+\frac{1}{q_{2}} \int_{\Lambda_{u}^{c}}|t|^{q_{2}} d \mu
$$

(where $\Lambda_{u}$ is defined in (2.1)), so that $u \in L^{\Phi}(\Omega)$ if and only if $\mu\left(\Lambda_{u}\right)<+\infty$ and $u \in L^{q_{1}}\left(\Lambda_{u}\right) \cap L^{q_{2}}\left(\Lambda_{u}^{c}\right)$. This is equivalent to $u \in L(\Omega)$, by Proposition 2.3.

As to the complementary function

$$
\Psi(t):=\sup _{s \geq 0}(|t| s-\Phi(s)) \quad \text { for all } t \in \mathbb{R}
$$

and the corresponding Orlicz class

$$
L^{\Psi}(\Omega)=\left\{\varphi \in \mathcal{M}(\Omega): \int_{\Omega} \Psi(\varphi) d \mu<+\infty\right\},
$$

an easy computation shows that $\Psi$ is actually given by

$$
\Psi(t)=\max _{s \geq 0}(|t| s-\Phi(s))= \begin{cases}\frac{1}{q_{2}^{\prime}}|t|^{q_{2}^{\prime}} & \text { if }|t| \leq 1 \\ \frac{1}{q_{1}^{\prime}}\left(|t|^{q_{1}^{\prime}}-1\right)+\frac{1}{q_{2}^{\prime}} & \text { if }|t|>1\end{cases}
$$

(where $q_{i}^{\prime}=q_{i} /\left(q_{i}-1\right)$ as usual), i.e.,

$$
\Psi(t)=\int_{0}^{|t|} \max \left\{s^{q_{1}^{\prime}-1}, s^{q_{2}^{\prime}-1}\right\} d s \quad \text { for all } t \in \mathbb{R},
$$

so that the same argument of the proof of Proposition 2.22 yields that

$$
L^{\Psi}(\Omega)=L^{q_{1}^{\prime}}(\Omega) \cap L^{q_{2}^{\prime}}(\Omega) .
$$

We now show that the Orlicz norm

$$
\|u\|_{\Phi}:=\sup \left\{\int_{\Omega}|u \varphi| d \mu: \varphi \in L^{\Psi}(\Omega), \int_{\Omega} \Psi(\varphi) d \mu \leq 1\right\}
$$

gives rise on $L(\Omega)$ to the same Banach structure we have considered so far. Recall the definition $(2.8)$ of $\|\cdot\|_{L^{q_{1}^{\prime}} \cap L^{q_{2}^{\prime}}}$.

Lemma 2.23. There exists $\delta_{0}>0$ such that for every $\varphi \in L^{\Psi}(\Omega)=L^{q_{1}^{\prime}}(\Omega) \cap$ $L^{q_{2}^{\prime}}(\Omega)$ one has

$$
\|\varphi\|_{L^{q_{1}^{\prime} \cap L^{q_{2}^{\prime}}}} \leq \delta_{0} \Longrightarrow \int_{\Omega} \Psi(\varphi) d \mu \leq 1 .
$$


Proof. Since

$$
\int_{\Lambda_{\varphi}^{c}}|\varphi|^{q_{2}^{\prime}} d \mu \leq \int_{\Omega}|\varphi|^{q_{2}^{\prime}} d \mu \leq\|\varphi\|_{L^{q_{1}^{\prime} \cap L^{q_{2}^{\prime}}}}^{q^{\prime}}
$$

and

$$
\mu\left(\Lambda_{\varphi}\right) \leq \int_{\Lambda_{\varphi}}|\varphi|^{q_{1}^{\prime}} d \mu \leq \int_{\Omega}|\varphi|^{q_{1}^{\prime}} d \mu \leq\|\varphi\|_{L_{1}^{q_{1}^{\prime} \cap L^{q_{2}^{\prime}}}}^{q^{\prime}}
$$

(recall definition (2.1)), from (2.25) we get

$$
\begin{aligned}
& \int_{\Omega} \Psi(\varphi) d \mu=\frac{1}{q_{1}^{\prime}} \int_{\Lambda_{\varphi}}|\varphi|^{q_{1}^{\prime}} d \mu+\left(\frac{1}{q_{2}^{\prime}}-\frac{1}{q_{1}^{\prime}}\right) \mu\left(\Lambda_{\varphi}\right)+\frac{1}{q_{2}^{\prime}} \int_{\Lambda_{\varphi}^{c}}|\varphi|^{q_{2}^{\prime}} d \mu \\
& \leq \frac{1}{q_{2}^{\prime}}\left(\|\varphi\|_{L_{1}^{q_{1}^{\prime} \cap L^{q_{2}^{\prime}}}}^{q^{\prime}}+\|\varphi\|_{L_{1}^{q_{1}^{\prime} \cap L^{q_{2}^{\prime}}}}^{q^{\prime}}\right)
\end{aligned}
$$

(recall that $q_{2}^{\prime} \leq q_{1}^{\prime}$ ) and the result ensues.

Proposition 2.24. The Orlicz norm (2.26) is equivalent to (2.4).

Proof. Let $u \in L^{\Phi}(\Omega)=L(\Omega)$. For any $\varphi \in L^{\Psi}(\Omega), \varphi \neq 0$, the mapping

$$
\widetilde{\varphi}:=\delta_{0} \frac{\varphi}{\|\varphi\|_{L_{1}^{q_{1}^{\prime}}} \in L^{q_{2}^{\prime}}} \in L^{\Psi}(\Omega),
$$

where $\delta_{0}$ is given by Lemma 2.23 , satisfies $\int_{\Omega} \Psi(\widetilde{\varphi}) d \mu \leq 1$ by $(2.27)$, so that we get

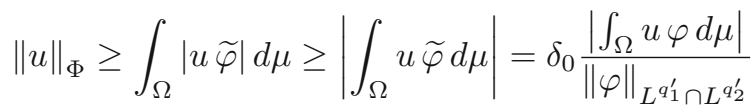

by (2.26). Hence (2.9) yields

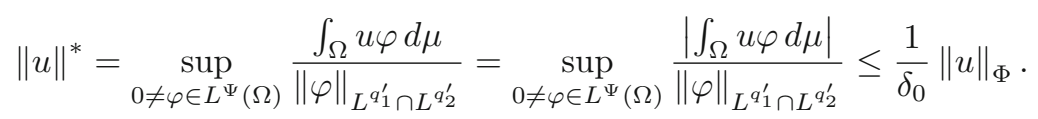

On the other hand, since $q_{2}^{\prime} \leq q_{1}^{\prime}$, for every $\varphi \in L^{\Psi}(\Omega)$ we have

$$
\int_{\Lambda_{\varphi}}|\varphi|^{q_{1}^{\prime}} d \mu \geq \int_{\Lambda_{\varphi}}|\varphi|^{q_{2}^{\prime}} d \mu \quad \text { and } \quad \int_{\Lambda_{\varphi}^{c}}|\varphi|^{q_{2}^{\prime}} d \mu \geq \int_{\Lambda_{\varphi}^{c}}|\varphi|^{q_{1}^{\prime}} d \mu
$$

(recall definition (2.1)), so that, using (2.25), we get

$$
\begin{aligned}
\int_{\Omega} \Psi(\varphi) d \mu & =\frac{1}{q_{1}^{\prime}} \int_{\Lambda_{\varphi}}|\varphi|^{q_{1}^{\prime}} d \mu+\left(\frac{1}{q_{2}^{\prime}}-\frac{1}{q_{1}^{\prime}}\right) \mu\left(\Lambda_{\varphi}\right)+\frac{1}{q_{2}^{\prime}} \int_{\Lambda_{\varphi}^{c}}|\varphi|^{q_{2}^{\prime}} d \mu \\
& \geq \frac{1}{q_{1}^{\prime}}\left(\int_{\Lambda_{\varphi}}|\varphi|^{q_{1}^{\prime}} d \mu+\int_{\Lambda_{\varphi}^{c}}|\varphi|^{q_{2}^{\prime}} d \mu\right) \\
& \geq \frac{1}{q_{1}^{\prime}} \max \left\{\int_{\Omega}|\varphi|^{q_{1}^{\prime}} d \mu, \int_{\Omega}|\varphi|^{q_{2}^{\prime}} d \mu\right\} .
\end{aligned}
$$

Hence, by Lemma $2.9, \int_{\Omega} \Psi(\varphi) d \mu \leq 1$ implies

$$
\int_{\Omega}|u \varphi| d \mu \leq\left(\|\varphi\|_{L^{q_{1}^{\prime}(\Omega)}}+\|\varphi\|_{L_{2}^{q_{2}^{\prime}(\Omega)}}\right)\|u\|^{*} \leq\left(\left(q_{1}^{\prime}\right)^{1 / q_{1}^{\prime}}+\left(q_{1}^{\prime}\right)^{1 / q_{2}^{\prime}}\right)\|u\|^{*}
$$


and we conclude

$$
\|u\|_{\Phi} \leq\left(\left(q_{1}^{\prime}\right)^{1 / q_{1}^{\prime}}+\left(q_{1}^{\prime}\right)^{1 / q_{2}^{\prime}}\right)\|u\|^{*}
$$

by (2.26). This completes the proof.

\section{The Nemytskiul operator on $L(\Omega)$}

As in the previous section, we fix $1<q_{1} \leq q_{2}<\infty$ and let $(\Omega, \mathcal{A}, \mu)$ be a nonempty $\sigma$-finite measure space, on which we also consider here a second measure $\lambda$, possibly not different from $\mu$, such that $\mu$ and $\lambda$ are absolutely continuous with respect to each other, that is,

$$
d \mu=\omega(x) d \lambda \text { for some measurable function } \omega: \Omega \rightarrow(0,+\infty) .
$$

Accordingly, we use the expanded notation $L^{p}(\Omega, d \lambda)$ for the Lebesgue spaces with respect to the measure $\lambda$, while we still briefly denote

$L^{p}:=L^{p}(\Omega, d \mu), \quad L:=L^{q_{1}}+L^{q_{2}}=L^{q_{1}}(\Omega, d \mu)+L^{q_{2}}(\Omega, d \mu)=: L(\Omega, d \mu)$.

Notice that a proposition holds $\mu$-a.e. if and only if it holds $\lambda$-a.e., since $\mu$ and $\lambda$ have the same null measure sets.

Theorem 3.1. Let $1 \leq q<\infty$ and let $f: \Omega \times \mathbb{R} \rightarrow \mathbb{R}$ be a Caratheodory function such that

$$
\int_{\Omega}|f(x, u(x))|^{q} d \lambda<+\infty \quad \text { for all } u \in L(\Omega, d \mu) .
$$

Then the operator

$$
\begin{gathered}
\mathcal{N}: L(\Omega, d \mu) \rightarrow L^{q}(\Omega, d \lambda) \\
\mathcal{N}(u)(x):=f(x, u(x))
\end{gathered}
$$

is continuous.

The proof of Theorem 3.1 will be achieved through several lemmas.

Lemma 3.2. Let $\left\{u_{n}\right\}$ be such that $u_{n} \rightarrow 0$ in $L$. Then, up to a subsequence, there exist $\left\{u_{n}^{\prime}\right\} \subseteq L^{q_{1}}$ and $\left\{u_{n}^{\prime \prime}\right\} \subseteq L^{q_{2}}$ such that

$$
u_{n}=u_{n}^{\prime}+u_{n}^{\prime \prime}, \quad \sum_{n=1}^{\infty} \int_{\Omega}\left|u_{n}^{\prime}\right|^{q_{1}} d \mu<+\infty, \quad \sum_{n=1}^{\infty} \int_{\Omega}\left|u_{n}^{\prime \prime}\right|^{q_{2}} d \mu<+\infty .
$$

Proof. Let $\left\{\varepsilon_{n}\right\}$ be an arbitrary sequence of real numbers such that

$$
\sum_{n=1}^{\infty} \varepsilon_{n}<+\infty
$$

Since $u_{n} \rightarrow 0$ in $L$, for all $n$ there exists $k_{n} \in \mathbb{N}$ such that $\left\|u_{k_{n}}\right\|<\min \left\{\varepsilon_{n}^{1 / q_{1}}\right.$, $\left.\varepsilon_{n}^{1 / q_{2}}\right\}$, and thus, by definition (2.2) of $\|\cdot\|$, there exist $u_{k_{n}}^{\prime} \in L^{q_{1}}$ and $u_{k_{n}}^{\prime \prime} \in L^{q_{2}}$ such that

$$
u_{k_{n}}=u_{k_{n}}^{\prime}+u_{k_{n}}^{\prime \prime} \quad \text { and } \quad\left\|u_{k_{n}}^{\prime}\right\|_{L^{q_{1}}}+\left\|u_{k_{n}}^{\prime \prime}\right\|_{L^{q_{2}}}<\min \left\{\varepsilon_{n}^{1 / q_{1}}, \varepsilon_{n}^{1 / q_{2}}\right\} .
$$

Together with (3.5), this gives the result. 
Lemma 3.3. Let $1 \leq q<\infty$ and let $f_{0}: \Omega \times \mathbb{R} \rightarrow \mathbb{R}$ be a Caratheodory function such that $f_{0}(\cdot, 0)=0$ and $f_{0}(\cdot, u(\cdot)) \in L^{q}(\Omega, d \lambda)$ for all $u \in L(\Omega, d \mu)$. Then the operator

$$
\begin{aligned}
& \mathcal{N}_{0}: L(\Omega, d \mu) \rightarrow L^{q}(\Omega, d \lambda) \\
& \mathcal{N}_{0}(u)(x):=f_{0}(x, u(x))
\end{aligned}
$$

is continuous at 0 .

Proof. First, we notice that $\mathcal{N}_{0}$ is well defined and $\mathcal{N}_{0}(0)=0$. Then, arguing by contradiction, we assume that $\mathcal{N}_{0}$ is not continuous at 0 , that is, there exist $\delta>0$ and a sequence $\left\{u_{n}\right\} \subseteq L$ such that $u_{n} \rightarrow 0$ in $L$ and

$$
\left\|\mathcal{N}_{0}\left(u_{n}\right)\right\|_{L^{q}(\Omega, d \lambda)}>\delta \text { for all } n
$$

that is,

$$
\int_{\Omega} \frac{\left|f_{0}\left(x, u_{n}(x)\right)\right|^{q}}{\omega(x)} d \mu=\int_{\Omega}\left|f_{0}\left(x, u_{n}(x)\right)\right|^{q} d \lambda>\delta^{q} \quad \text { for all } n .
$$

Passing in case to a subsequence, let $\left\{u_{n}^{\prime}\right\} \subseteq L^{q_{1}}$ and $\left\{u_{n}^{\prime \prime}\right\} \subseteq L^{q_{2}}$ be such that (3.4) holds according to Lemma 3.2.

We claim that $\forall k \in \mathbb{N}$ there exist $D_{k} \subseteq \Omega$ and $n_{k} \in \mathbb{N}$ such that

$$
\begin{gathered}
\mu\left(D_{k}\right)<+\infty \\
D_{k} \cap D_{k^{\prime}}=\varnothing \quad \text { if } k \neq k^{\prime} \\
\int_{D_{k}} \frac{\left|f_{0}\left(x, u_{n_{k}}(x)\right)\right|^{q}}{\omega(x)} d \mu>\frac{\delta^{q}}{2} .
\end{gathered}
$$

Let us proceed by induction on $k$. For $k=0$, we set $n_{0}=0$, so that (3.7) and the $\sigma$-finiteness of $\mu$ imply that $\exists D_{0} \subseteq \Omega$ such that $\mu\left(D_{0}\right)<+\infty$ and

$$
\int_{D_{0}} \frac{\left|f_{0}\left(x, u_{n_{0}}(x)\right)\right|^{q}}{\omega(x)} d \mu>\delta^{q}>\frac{\delta^{q}}{2} .
$$

Then suppose that we have $D_{0}, \ldots, D_{k} \subseteq \Omega$ and $n_{0}, \ldots, n_{k} \in \mathbb{N}$ satisfying (3.8)-(3.10), and define the set

$$
D:=\bigcup_{j=0}^{k} D_{j}
$$

Since $\mu(D)<+\infty$, by Proposition 2.17 we know that $u_{n} \rightarrow 0$ in $L^{q_{1}}(D, d \mu)$ and we can apply the classical theorem on Nemytskil operators (see [36, Theorem 19.1]): the operator

$$
\begin{gathered}
\widetilde{\mathcal{N}}_{0}: L^{q_{1}}(D, d \mu) \rightarrow L^{q}(D, d \mu) \\
\widetilde{\mathcal{N}}_{0}(u)(x):=\omega(x)^{-1 / q} f_{0}(x, u(x))
\end{gathered}
$$

(associated to the Caratheodory function $\widetilde{f}_{0}(x, t):=\omega(x)^{-1 / q} f_{0}(x, t)$ ) is continuous and such that $\widetilde{\mathcal{N}}_{0}(0)=0$, and thus there exists $n_{k+1} \in \mathbb{N}, n_{k+1}>n_{k}$, such that

$$
\int_{D} \frac{\left|f_{0}\left(x, u_{n_{k+1}}(x)\right)\right|^{q}}{\omega(x)} d \mu<\frac{\delta^{q}}{2}
$$


On the other hand, again by (3.7) and the $\sigma$-finiteness of $\mu$, there exists $D^{\prime} \subseteq \Omega$ such that $\mu\left(D^{\prime}\right)<+\infty$ and

$$
\int_{D^{\prime}} \frac{\left|f_{0}\left(x, u_{n_{k+1}}(x)\right)\right|^{q}}{\omega(x)} d \mu>\delta^{q} .
$$

Then, setting

$$
D_{k+1}=D^{\prime} \backslash D
$$

from (3.11)-(3.12) we deduce

$$
\int_{D_{k+1}} \frac{\left|f_{0}\left(x, u_{n_{k+1}}(x)\right)\right|^{q}}{\omega(x)} d \mu>\frac{\delta^{q}}{2}
$$

and the claim is proved.

Now, using (3.9), we can define

$$
u^{\prime}:=\sum_{k=0}^{\infty} u_{n_{k}}^{\prime} \chi_{D_{k}}
$$

and, by (3.4), we get

$$
\int_{\Omega}\left|u^{\prime}\right|^{q_{1}} d \mu=\sum_{k=0}^{\infty} \int_{D_{k}}\left|u_{n_{k}}^{\prime}\right|^{q_{1}} d \mu \leq \sum_{k=0}^{\infty} \int_{\Omega}\left|u_{n_{k}}^{\prime}\right|^{q_{1}} d \mu<+\infty
$$

so that $u^{\prime} \in L^{q_{1}}$. Analogously, one defines $u^{\prime \prime} \in L^{q_{2}}$ by setting

$$
u^{\prime \prime}:=\sum_{k=0}^{\infty} u_{n_{k}}^{\prime \prime} \chi_{D_{k}} .
$$

Therefore $u:=u^{\prime}+u^{\prime \prime}$ belongs to $L$ and satisfies

$$
u=\sum_{k=0}^{\infty}\left(u_{n_{k}}^{\prime}+u_{n_{k}}^{\prime \prime}\right) \chi_{D_{k}}=\sum_{k=0}^{\infty} u_{n_{k}} \chi_{D_{k}},
$$

which, by $f_{0}(\cdot, 0)=0$ and $(3.10)$, implies

$$
\begin{aligned}
\int_{\Omega}\left|f_{0}(x, u(x))\right|^{q} d \lambda & =\int_{\Omega} \frac{\left|f_{0}(x, u(x))\right|^{q}}{\omega(x)} d \mu=\sum_{k=0}^{\infty} \int_{D_{k}} \frac{\left|f_{0}\left(x, u_{n_{k}}(x)\right)\right|^{q}}{\omega(x)} d \mu \\
& =+\infty .
\end{aligned}
$$

So we get a contradiction with $f_{0}(\cdot, u(\cdot)) \in L^{q}(\Omega, d \lambda)$.

Proof of Theorem 3.1. Let $u_{0} \in L$ and define

$$
f_{0}(x, t):=f\left(x, t+u_{0}(x)\right)-f\left(x, u_{0}(x)\right)
$$

(for almost every $x \in \Omega$ and every $t \in \mathbb{R}$ ). Then the operator $\mathcal{N}_{0}: L \rightarrow$ $L^{q}(\Omega, d \lambda)$ defined by (3.6) is continuous at 0 thanks to Lemma 3.3, and therefore $\mathcal{N}$ is continuous at $u_{0}$ since $\mathcal{N}(u)-\mathcal{N}\left(u_{0}\right)=\mathcal{N}_{0}\left(u-u_{0}\right)$.

We now introduce the following condition: 
( $\boldsymbol{\mu})$ for every $v \in L^{1}(\Omega, d \mu)$ and $n \geq 1$ there exists a partition $\left\{\Omega_{i}\right\}_{1 \leq i \leq n}$ of $\Omega$ such that

$$
\int_{\Omega_{i}}|v| d \mu=\frac{1}{n} \int_{\Omega}|v| d \mu \quad \text { for all } i=1, \ldots, n .
$$

We observe that $(\boldsymbol{\mu})$ holds for example if $\Omega \subseteq \mathbb{R}^{N}$ and $d \mu=\omega(x) d x$ for some $\omega \in L_{\text {loc }}^{1}(\Omega, d x)$; indeed, in this case, the mapping the mapping $\varphi: \rho \mapsto$ $\int_{\Omega \cap B_{\rho}}|v| d \mu$ is continuous, monotone and such that $\varphi([0,+\infty))=[0, \alpha]$ where $\alpha:=\int_{\Omega}|v| d \mu$, so that, setting $\rho_{i}:=\varphi^{-1}(\alpha i / n)$ and $\Omega_{i}:=\Omega \cap\left(B_{\rho_{i}} \backslash B_{\rho_{i-1}}\right)$ for $i=1, \ldots, n$, one has

$$
\int_{\Omega_{i}}|v| d \mu=\int_{\Omega \cap B_{\rho_{i}}}|v| d \mu-\int_{\Omega \cap B_{\rho_{i-1}}}|v| d \mu=\frac{\alpha i}{n}-\frac{\alpha(i-1)}{n}=\frac{\alpha}{n} .
$$

Theorem 3.4. If $(\boldsymbol{\mu})$ holds then, under the same assumptions of Theorem 3.1 , the operator (3.2)-(3.3) is bounded (i.e., it maps bounded sets into bounded sets).

Proof. Define

$$
f_{0}(x, t):=f(x, t)-f(x, 0) \quad \text { for all }(x, t) \in \Omega \times \mathbb{R},
$$

so that the operator $\mathcal{N}_{0}: L \rightarrow L^{q}(\Omega, d \lambda)$ defined by (3.6) is continuous at 0 thanks to Lemma 3.3. Note that

$$
\mathcal{N}_{0}(u)=\mathcal{N}(u)-\mathcal{N}(0)
$$

and thus $\mathcal{N}_{0}(0)=0$. Hence there exists $R>0$ such that $\forall u \in L$ one has

$$
\|u\| \leq R \Longrightarrow\left\|\mathcal{N}_{0}(u)\right\|_{L^{q}(\Omega, d \lambda)} \leq 1
$$

Now let $u \in L$ and let $n \in \mathbb{N}$ be such that

$$
n^{1 / q_{2}} \leq\left\|\frac{2 u}{R}\right\| \leq(n+1)^{1 / q_{2}}
$$

By definition (2.2) of $\|\cdot\|$, there exist $u^{\prime} \in L^{q_{1}}$ and $u^{\prime \prime} \in L^{q_{2}}$ such that

$$
\begin{aligned}
\frac{2 u}{R} & =\frac{2 u^{\prime}}{R}+\frac{2 u^{\prime \prime}}{R}, \\
n^{1 / q_{2}} & \leq\left\|\frac{2 u^{\prime}}{R}\right\|_{L^{q_{1}}}+\left\|\frac{2 u^{\prime \prime}}{R}\right\|_{L^{q_{2}}}<(n+1)^{1 / q_{2}},
\end{aligned}
$$

whence we get

$$
\left\|\frac{2 u^{\prime}}{R}\right\|_{L^{q_{1}}}<(n+1)^{1 / q_{2}} \leq(n+1)^{1 / q_{1}} \text { and }\left\|\frac{2 u^{\prime \prime}}{R}\right\|_{L^{q_{2}}}<(n+1)^{1 / q_{2}},
$$

that is,

$$
\int_{\Omega}\left|u^{\prime}\right|^{q_{1}} d \mu<(n+1)\left(\frac{R}{2}\right)^{q_{1}} \text { and } \int_{\Omega}\left|u^{\prime \prime}\right|^{q_{2}} d \mu<(n+1)\left(\frac{R}{2}\right)^{q_{2}} .
$$

Hence, by assumption $(\boldsymbol{\mu})$, there exist two partitions $\left\{A_{i}\right\},\left\{B_{i}\right\}, 1 \leq i \leq n+1$, of $\Omega$ such that for all $i=1, \ldots, n+1$ one has

$$
\int_{A_{i}}\left|u^{\prime}\right|^{q_{1}} d \mu<\left(\frac{R}{2}\right)^{q_{1}} \text { and } \int_{B_{i}}\left|u^{\prime \prime}\right|^{q_{2}} d \mu<\left(\frac{R}{2}\right)^{q_{2}} .
$$


Define a new partition $\left\{C_{i j}\right\}$ of $\Omega$ by setting

$$
C_{i j}:=A_{i} \cap B_{j} \quad \text { for } i, j=1, \ldots, n+1 .
$$

Then $u^{\prime} \in L^{q_{1}}\left(C_{i j}, d \mu\right), u^{\prime \prime} \in L^{q_{2}}\left(C_{i j}, d \mu\right)$ and

$$
\left\|u^{\prime}\right\|_{L^{q_{1}}\left(C_{i j}, d \mu\right)},\left\|u^{\prime \prime}\right\|_{L^{q_{2}}\left(C_{i j}, d \mu\right)}<\frac{R}{2},
$$

so that the mapping defined by

$$
u_{i j}(x):= \begin{cases}u(x) & \text { if } x \in C_{i j} \\ 0 & \text { otherwise }\end{cases}
$$

belongs to $L$ by Proposition 2.3 and satisfies

$$
\left\|u_{i j}\right\| \leq\left\|u^{\prime}\right\|_{L^{q_{1}}\left(C_{i j}, d \mu\right)}+\left\|u^{\prime \prime}\right\|_{L^{q_{2}}\left(C_{i j}, d \mu\right)}<R,
$$

since (3.16) implies $u_{i j}=u^{\prime} \chi_{C_{i j}}+u^{\prime \prime} \chi_{C_{i j}}$. Hence (3.14) gives

$$
\left\|\mathcal{N}_{0}\left(u_{i j}\right)\right\|_{L^{q}(\Omega, d \lambda)} \leq 1,
$$

and thus we get

$$
\begin{aligned}
\left\|\mathcal{N}_{0}(u)\right\|_{L^{q}(\Omega, d \lambda)}^{q} & =\int_{\Omega}\left|f_{0}(x, u)\right|^{q} d \lambda=\sum_{i . j=1}^{n+1} \int_{C_{i j}}\left|f_{0}(x, u)\right|^{q} d \lambda \\
& =\sum_{i . j=1}^{n+1} \int_{\Omega}\left|f_{0}\left(x, u_{i j}\right)\right|^{q} d \lambda=\sum_{i . j=1}^{n+1}\left\|\mathcal{N}_{0}\left(u_{i j}\right)\right\|_{L^{q}(\Omega, d \lambda)}^{q} \\
& \leq(n+1)^{2} .
\end{aligned}
$$

Therefore, by (3.13) and the first inequality of (3.15), we deduce

$$
\|\mathcal{N}(u)-\mathcal{N}(0)\|_{L^{q}(\Omega, d \lambda)}^{q}=\left\|\mathcal{N}_{0}(u)\right\|_{L^{q}(\Omega, d \lambda)}^{q} \leq\left(\left(\frac{2}{R}\right)^{q_{2}}\|u\|^{q_{2}}+1\right)^{2} .
$$

This yields the result and the proof is thus complete.

A growth condition on $f$ ensuring (3.1) can be easily obtained by Proposition 2.3.

Proposition 3.5. Let $f: \Omega \times \mathbb{R} \rightarrow \mathbb{R}$ be a Caratheodory function and let $\alpha, \beta>0$ be such that

$$
\frac{\alpha}{\beta} \leq \frac{q_{1}}{q_{2}} \quad \text { and } \quad \beta \leq q_{2} .
$$

Assume that there exist $q \in\left[q_{2} / \beta, q_{1} / \alpha\right]$ and $g \in L^{q}(\Omega, d \mu)$ such that for almost every $x \in \Omega$ and every $t \in \mathbb{R}$ one has

$$
|f(x, t)| \leq\left(M \min \left\{|t|^{\alpha},|t|^{\beta}\right\}+g(x)\right) \omega(x)^{1 / q}
$$

where $M>0$ is a constant. Then the Nemytskiu operator $\mathcal{N}: L(\Omega, d \mu) \rightarrow$ $L^{q}(\Omega, d \lambda)$ given by (3.3) is well defined and continuous, and it is bounded if ( $\boldsymbol{\mu})$ holds. 
Proof. By Theorems 3.1 and 3.4, it is sufficient to show that $f(\cdot, u(\cdot)) \in$ $L^{q}(\Omega, d \lambda)$ for all $u \in L$. So let $u \in L$ and recall the definition (2.1) of $\Lambda_{u}$. Since $\alpha \leq \beta$, we have $|f(\cdot, u(\cdot))| \leq\left(M|u|^{\alpha} \chi_{\Lambda_{u}}+M|u|^{\beta} \chi_{\Lambda_{u}^{c}}+g\right) \omega^{1 / q}$ almost everywhere on $\Omega$, so that there exists a constant $C>0$ such that

$$
\begin{aligned}
\int_{\Omega}|f(x, u)|^{q} d \lambda & \leq \int_{\Omega}\left(M|u|^{\alpha} \chi_{\Lambda_{u}}+M|u|^{\beta} \chi_{\Lambda_{u}^{c}}+g\right)^{q} \omega(x) d \lambda \\
& \leq C\left(\int_{\Lambda_{u}}|u|^{\alpha q} d \mu+\int_{\Lambda_{u}^{c}}|u|^{\beta q} d \mu+\int_{\Omega} g^{q} d \mu\right) \\
& \leq C\left(\int_{\Lambda_{u}}|u|^{q_{1}} d \mu+\int_{\Lambda_{u}^{c}}|u|^{q_{2}} d \mu+\|g\|_{L^{q}}^{q}\right),
\end{aligned}
$$

where we have used the fact that $\alpha q \leq q_{1}$ and $\beta q \geq q_{2}$. The conclusion then follows from Proposition 2.3.iii.

Remark 3.6. If $\mu=\lambda$, then Proposition 3.5 ensures that the operator $\mathcal{N}$ : $L \rightarrow L^{q}$ given by (3.3) is continuous (and bounded if $(\boldsymbol{\mu})$ holds) for every $q \in$ $\left[q_{2} / \beta, q_{1} / \alpha\right]$, provided that (3.17) holds with $\omega(x) \equiv 1$ and $g \in L^{q_{1} / \alpha} \cap L^{q_{2} / \beta}$. Taking into account the characterization of Proposition 2.22, such a result was partially given in [25, Theorem 2.3], where it is shown that a necessary and sufficient condition in order that $\mathcal{N}$ acts from $L^{\Phi}$ into $L^{q}$ is that there exist $\tilde{M}>0$ and $\tilde{g} \in L^{1}$ such that

$$
|f(x, t)|^{q} \leq \tilde{M} \Phi(t)+\tilde{g}(x) \quad \text { for almost every } x \in \Omega \text { and every } t \in \mathbb{R} \text {. }
$$

Indeed, under the assumptions of Proposition 3.5, it is easy to check that there exists a constant $C>0$ such that $\min \left\{|t|^{\alpha},|t|^{\beta}\right\}^{q} \leq C \Phi(t)$ for all $t \in \mathbb{R}$, so that (3.18) holds provided that (3.17) holds.

The following corollary concerns the case, of particular interest in the applications, in which the Nemytskiu operator works between $L=L(\Omega, d \mu)$ and its dual space $L^{\prime}$. Recall from Theorem 2.10 that $L^{\prime}$ identifies with $L^{q_{1}^{\prime}} \cap$ $L^{q_{2}^{\prime}}=L^{q_{1}^{\prime}}(\Omega, d \mu) \cap L^{q_{2}^{\prime}}(\Omega, d \mu)$.

Corollary 3.7. Let $f: \Omega \times \mathbb{R} \rightarrow \mathbb{R}$ be a Caratheodory function and assume that there exist $M>0$ and $g \in L^{q_{1}^{\prime}} \cap L^{q_{2}^{\prime}}$ such that for almost every $x \in \Omega$ and every $t \in \mathbb{R}$ one has

$$
|f(x, t)| \leq M \min \left\{|t|^{q_{1}-1},|t|^{q_{2}-1}\right\}+g(x) .
$$

Then the Nemytskiu operator $\mathcal{N}: L \rightarrow L^{q_{1}^{\prime}} \cap L^{q_{2}^{\prime}}$ given by (3.3) is well defined and continuous, and it is bounded if $(\boldsymbol{\mu})$ holds.

Proof. Recalling the intersection norm (2.8), the continuity and the boundedness of $\mathcal{N}: L(\Omega) \rightarrow L^{q_{1}^{\prime}} \cap L^{q_{2}^{\prime}}$ is equivalent to the ones of $\mathcal{N}$ from $L$ into both $L^{q_{1}^{\prime}}$ and $L^{q_{2}^{\prime}}$. On the other hand, $f$ satisfies (3.17) with $\alpha=q_{1}-1$, $\beta=q_{2}-1, \omega(x) \equiv 1$ and $g \in L^{q_{1}^{\prime}} \cap L^{q_{2}^{\prime}}$. Hence the result follows from applying Proposition 3.5 with $q=q_{1}^{\prime}, q_{2}^{\prime}$. 
Another consequence of Theorem 3.1 (and in particular of Corollary 3.7) is the next differentiability result, which will be exploited in Sect. 5 .

Proposition 3.8. Let $f: \Omega \times \mathbb{R} \rightarrow \mathbb{R}$ be a Caratheodory function and set

$$
F(x, t):=\int_{0}^{t} f(x, s) d s \quad \text { for all }(x, t) \in \Omega \times \mathbb{R} .
$$

Assume that there exist $M>0$ and $g \in L^{q_{1}^{\prime}} \cap L^{q_{2}^{\prime}}$ such that for almost every $x \in \Omega$ and every $t \in \mathbb{R}$ one has

$$
|f(x, t)| \leq\left(M \min \left\{|t|^{q_{1}-1},|t|^{q_{2}-1}\right\}+g(x)\right) \omega(x) .
$$

Then the operator

$$
\begin{gathered}
\mathcal{F}: L(\Omega, d \mu) \rightarrow L^{1}(\Omega, d \lambda) \\
\mathcal{F}(u)(x):=F(x, u(x))
\end{gathered}
$$

is well defined and of class $C^{1}$, with Fréchet derivative $\mathcal{F}^{\prime}(u)$ at any $u \in$ $L(\Omega, d \mu)$ given by

$$
\left\langle\mathcal{F}^{\prime}(u), h\right\rangle(x)=f(x, u(x)) h(x) \quad \text { for all } h \in L(\Omega, d \mu) .
$$

Proof. From (3.19) it follows that $\exists \tilde{M}>0$ such that

$$
|F(x, t)| \leq\left(\tilde{M} \min \left\{|t|^{q_{1}},|t|^{q_{2}}\right\}+|t| g(x)\right) \omega(x)
$$

(for almost every $x \in \Omega$ and every $t \in \mathbb{R}$ ), so that $u \in L$ implies

$$
|\mathcal{F}(u)| \leq \tilde{M} \min \left\{|u|^{q_{1}},|u|^{q_{2}}\right\} \omega+|u| g \omega \in L^{1}(\Omega, d \lambda)
$$

since

$$
\begin{aligned}
\int_{\Omega} \min \left\{|u|^{q_{1}},|u|^{q_{2}}\right\} \omega d \lambda & =\int_{\Omega} \min \left\{|u|^{q_{1}},|u|^{q_{2}}\right\} d \mu<+\infty \\
\int_{\Omega}|u| g \omega d \lambda & =\int_{\Omega}|u| g d \mu<+\infty
\end{aligned}
$$

by Proposition 3.5 and Lemma 2.9 respectively. Hence the operator (3.20) is well defined.

Now let $u, h \in L$ and let $\left\{t_{n}\right\} \subseteq(-1,1)$ be any sequence such that $t_{n} \rightarrow 0$. By the mean value theorem, we infer that for almost every $x \in \Omega$ and every $n$ there exists $\theta_{n}=\theta_{n}(x) \in[0,1]$ such that

$$
\begin{aligned}
& \left|F\left(x, u+t_{n} h\right)-F(x, u)\right| \\
& \quad=\left|f\left(x, u+\theta_{n} t_{n} h\right)\right|\left|t_{n}\right||h| \\
& \quad \leq\left(M \min \left\{\left|u+\theta_{n} t_{n} h\right|^{q_{1}-1},\left|u+\theta_{n} t_{n} h\right|^{q_{2}-1}\right\}+g\right)\left|t_{n}\right||h| \omega \\
& \quad \leq\left(M \min \left\{(|u|+|h|)^{q_{1}-1},(|u|+|h|)^{q_{2}-1}\right\}+g\right)\left|t_{n}\right||h| \omega,
\end{aligned}
$$

so that almost everywhere on $\Omega$ one has

$$
\frac{\mathcal{F}\left(u+t_{n} h\right)-\mathcal{F}(u)}{t_{n}} \rightarrow f(x, u) h \quad \text { as } n \rightarrow \infty
$$


and

$$
\begin{aligned}
\left|\frac{\mathcal{F}\left(u+t_{n} h\right)-\mathcal{F}(u)}{t_{n}}\right| \leq & M \min \left\{(|u|+|h|)^{q_{1}-1},(|u|+|h|)^{q_{2}-1}\right\}|h| \omega \\
& +g|h| \omega
\end{aligned}
$$

where

$$
\min \left\{(|u|+|h|)^{q_{1}-1}, \quad(|u|+|h|)^{q_{2}-1}\right\}|h| \omega, \quad g|h| \omega \in L^{1}(\Omega, d \lambda)
$$

by Lemma 2.9, since $|u|+|h| \in L$ (recall Proposition 2.3.iv) and Corollary 3.7 gives $\min \left\{(|u|+|h|)^{q_{1}-1},(|u|+|h|)^{q_{2}-1}\right\} \in L^{q_{1}^{\prime}} \cap L^{q_{2}^{\prime}}$. Hence

$$
\frac{\mathcal{F}\left(u+t_{n} h\right)-\mathcal{F}(u)}{t_{n}} \rightarrow f(x, u) h \quad \text { in } L^{1}(\Omega, d \lambda)
$$

by dominated convergence. On the other hand, the Caratheodory function defined by

$$
\widetilde{f}(x, t):=\frac{f(x, t)}{\omega(x)} \text { for all }(x, t) \in \Omega \times \mathbb{R}
$$

satisfies

$$
|\tilde{f}(x, t)|=\frac{|f(x, t)|}{\omega(x)} \leq M \min \left\{|t|^{q_{1}-1},|t|^{q_{2}-1}\right\}+g(x)
$$

(for almost every $x \in \Omega$ and every $t \in \mathbb{R}$ ), so that, by Corollary 3.7, the operator $u \mapsto \widetilde{f}(x, u)$ acts from $L$ into $L^{q_{1}^{\prime}} \cap L^{q_{2}^{\prime}}$ and it is continuous. Hence the linear operator

$$
\mathcal{F}^{\prime}(u): h \in L \mapsto f(x, u) h \in L^{1}(\Omega, d \lambda)
$$

is continuous by Lemma 2.9 and thus, by (3.21), it is the Gâteaux derivative of $\mathcal{F}$ at $u$. Moreover, denoting by $\mathcal{L}$ the space of linear and continuous operators from $L$ into $L^{1}(\Omega, d \lambda)$, for every $u, v \in L$ one has

$$
\begin{aligned}
\left\|\mathcal{F}^{\prime}(u)-\mathcal{F}^{\prime}(v)\right\|_{\mathcal{L}} & =\sup _{\|h\|=1} \int_{\Omega}|\widetilde{f}(x, u)-\widetilde{f}(x, v)||h| d \mu \\
& \leq\|\widetilde{f}(x, u)-\widetilde{f}(x, v)\|_{L^{q_{1}^{\prime} \cap L^{q_{2}^{\prime}}}}
\end{aligned}
$$

by Lemma 2.9 again, so that the mapping $u \in L \mapsto \mathcal{F}^{\prime}(u) \in \mathcal{L}$ is continuous and thus $\mathcal{F}^{\prime}(u)$ is the Fréchet derivative of $\mathcal{F}$ at $u$.

\section{A compactness result}

As a particular case of the previous sections, here we consider the space $L\left(\mathbb{R}^{N}, d \mu\right)$ where $\mu$ is a $\sigma$-finite Borel measure such that $\mu$ and the Lebesgue measure of $\mathbb{R}^{N}$ are absolutely continuous with respect to each other, that is,

$$
d \mu=\omega(x) d x \text { for some measurable function } \omega: \mathbb{R}^{N} \rightarrow(0,+\infty)
$$


Accordingly, we only omit the indication of the Lebesgue measure, briefly writing

$$
L^{p}(\Omega)=L^{p}(\Omega, d x) \quad \text { for any } \Omega \subseteq \mathbb{R}^{N},
$$

while, in order to exclude possible misunderstandings, whenever the measure $\mu$ is concerned we shall use expanded notations, that is,

$$
L\left(\mathbb{R}^{N}, \omega(x) d x\right)=L\left(\mathbb{R}^{N}, d \mu\right)=L^{q_{1}}\left(\mathbb{R}^{N}, d \mu\right)+L^{q_{2}}\left(\mathbb{R}^{N}, d \mu\right),
$$

just avoiding the mentioning of the exponents $q_{1}, q_{2}$ in the sum space notation.

Assuming $1<p<N$, we will prove a compactness result involving the radial subspace

$$
D_{\text {rad }}^{1, p}\left(\mathbb{R}^{N}\right):=\left\{u \in D^{1, p}\left(\mathbb{R}^{N}\right): u(x)=u(|x|)\right\}
$$

of the Sobolev space

$$
D^{1, p}\left(\mathbb{R}^{N}\right)=\left\{u \in L^{p^{*}}\left(\mathbb{R}^{N}\right):|\nabla u| \in L^{p}\left(\mathbb{R}^{N}\right)\right\},
$$

where $p^{*}:=p N /(N-p)$. Recall that $D^{1, p}\left(\mathbb{R}^{N}\right)$ is actually the completion of $C_{\mathrm{c}}^{\infty}\left(\mathbb{R}^{N}\right)$ with respect to the norm

$$
\|u\|_{D^{1, p}}:=\left(\int_{\mathbb{R}^{N}}|\nabla u|^{p} d x\right)^{1 / p}
$$

as well as $D_{\text {rad }}^{1, p}\left(\mathbb{R}^{N}\right)$ is the closure of the radial subspace $C_{\mathrm{c}, \text { rad }}^{\infty}\left(\mathbb{R}^{N}\right)$ of $C_{\mathrm{c}}^{\infty}\left(\mathbb{R}^{N}\right)$ in $D^{1, p}\left(\mathbb{R}^{N}\right)$. Moreover, the continuous embedding

$$
D^{1, p}\left(\mathbb{R}^{N}\right) \hookrightarrow L^{p^{*}}\left(\mathbb{R}^{N}\right)
$$

holds, thanks to Sobolev inequality.

Theorem 4.1. Assume $\omega \in L_{\text {loc }}^{\infty}\left(\mathbb{R}^{N} \backslash\{0\}\right)$ and assume that there exist $\theta_{1}>$ $-\left(1+N / p^{\prime}\right)$ and $\theta_{2} \in \mathbb{R}$ such that

$$
\lim _{r \rightarrow 0^{+}} \operatorname{essup}_{0<|x|<r} \frac{\omega(x)}{|x|^{\theta_{1}}}<+\infty \text { and } \lim _{r \rightarrow+\infty} \operatorname{ess\operatorname {sup}} \underset{|x|>r}{\operatorname{esc}} \frac{\omega(x)}{|x|^{\theta_{2}}}<+\infty \text {. }
$$

Then $D_{\text {rad }}^{1, p}\left(\mathbb{R}^{N}\right)$ is compactly embedded into $L\left(\mathbb{R}^{N}, \omega(x) d x\right)$ for every $q_{1}, q_{2}>1$ such that

$$
q_{1}<p^{*}+\frac{\theta_{1} p}{N-p} \text { and } p^{*}+\frac{\theta_{2} p}{N-p}<q_{2} .
$$

The proof of Theorem 4.1 relies on the following lemmas, where the first one is a generalization of a pointwise estimate which is well known for $p=2$. Some easy consequences of Theorem 4.1 will be pointed out at the end of the section.

Lemma 4.2. There exists a constant $C_{N, p}>0$ (only depending on $N, p$ ) such that $\forall u \in D_{\mathrm{rad}}^{1, p}\left(\mathbb{R}^{N}\right)$ one has

$$
|u(x)| \leq C_{N, p}\|u\|_{D^{1, p}} \frac{1}{|x|^{\frac{N-p}{p}}} \quad \text { almost everywhere on } \mathbb{R}^{N} .
$$

Proof. See for example [37, Lemma 1]. 
For future convenience, in the next two lemmas we give more general results than the ones needed in the proof of Theorem 4.1 (where we will apply with $h=u)$.

Lemma 4.3. Let $\theta>-\left(1+N / p^{\prime}\right)$ and $1<q<p^{*}+\theta p /(N-p)$. Then there exists $C_{0}=C_{0}(N, p, \theta, q)>0$ such that for every $u \in D_{\mathrm{rad}}^{1, p}\left(\mathbb{R}^{N}\right), h \in D^{1, p}\left(\mathbb{R}^{N}\right)$ and $r>0$ one has

$$
\int_{B_{r}}|x|^{\theta}|u|^{q-1}|h| d x \leq C_{0} r^{\left(p^{*}+\frac{\theta p}{N-p}-q\right) \frac{N-p}{p}}\|u\|_{D^{1, p}}^{q-1}\|h\|_{D^{1, p}} .
$$

Proof. We denote by $C$ any positive constant only depending on $N, p, \theta, q$. Take $\delta=\delta(N, p, q)>0$ such that $q-p^{*}<\delta<q-1$, in such a way that $\left(p^{*}-1\right) /(q-1-\delta)>1$. Then by Hölder inequality, Sobolev embedding (4.1) and Lemma 4.2 , for every $u \in D_{\text {rad }}^{1, p}\left(\mathbb{R}^{N}\right), h \in D^{1, p}\left(\mathbb{R}^{N}\right)$ and $r>0$ we have

$$
\begin{aligned}
& \int_{B_{r}}|x|^{\theta}|u|^{q-1}|h| d x \\
& \quad \leq\left(\int_{B_{r}}|x|^{\frac{\theta p^{*}}{p^{*}-1}}|u|^{\frac{(q-1) p^{*}}{p^{*}-1}} d x\right)^{\left(p^{*}-1\right) / p^{*}}\left(\int_{B_{r}}|h|^{p^{*}} d x\right)^{1 / p^{*}} \\
& \quad \leq\left(\int_{B_{r}}|x|^{\frac{\theta p^{*}}{p^{*}-1}}|u|^{\frac{\delta p^{*}}{p^{*}-1}}|u|^{\frac{(q-1-\delta) p^{*}}{p^{*}-1}} d x\right)^{\left(p^{*}-1\right) / p^{*}}\|h\|_{D^{1, p}} \\
& \leq C\|u\|_{D^{1, p}}^{\delta}\left(\int_{B_{r}}|x|^{\left(\theta-\frac{N-p}{p} \delta\right) \frac{p^{*}}{p^{*}-1}}|u|^{(q-1-\delta) p^{* \prime}} d x\right)^{1 / p^{* \prime}}\|h\|_{D^{1, p}}
\end{aligned}
$$

Applying Hölder inequality again (with conjugate exponents $t=\left(p^{*}-1\right) /(q-$ $1-\delta)$ and $\left.t^{\prime}=\left(p^{*}-1\right) /\left(p^{*}-q+\delta\right)\right)$ we get

$$
\begin{aligned}
& \int_{B_{r}}|x|^{\theta}|u|^{q-1}|h| d x \\
& \leq C\left(\int_{B_{r}}|x|^{\left(\theta-\frac{\delta N}{p^{*}}\right) \frac{p^{*}}{p^{*}-q+\delta}} d x\right)^{\frac{p^{*}-q+\delta}{p^{*}}}\left(\int_{B_{r}}|u|^{p^{*}} d x\right)^{\frac{q-1-\delta}{p^{*}}}\|u\|_{D^{1, p}}^{\delta}\|h\|_{D^{1, p}} \\
& \leq C\left(\int_{0}^{r} \rho^{\left(\theta-\frac{\delta N}{p^{*}}\right) \frac{p^{*}}{p^{*}-q+\delta}+N-1} d \rho\right)^{\left(p^{*}-q+\delta\right) / p^{*}}\|u\|_{D^{1, p}}^{q-1}\|h\|_{D^{1, p}}
\end{aligned}
$$

where

$$
\left(\theta-\frac{\delta N}{p^{*}}\right) \frac{p^{*}}{p^{*}-q+\delta}+N=\frac{N}{p^{*}-q+\delta}\left(p^{*}+\frac{\theta p}{N-p}-q\right)>0
$$

by assumption. Hence we conclude

$$
\int_{B_{r}}|x|^{\theta}|u|^{q-1}|h| d x \leq C\|u\|_{D^{1, p}}^{q}\left(r^{\frac{N}{p^{*}-q+\delta}}\left(p^{*}+\frac{\theta p}{N-p}-q\right)\right)^{\left(p^{*}-q+\delta\right) / p^{*}}
$$

which yields the result.

Lemma 4.4. Let $\theta \in \mathbb{R}$ and let $q>1$ be such that $q>p^{*}+\theta p /(N-p)$. Then there exists $C_{\infty}=C_{\infty}(N, p, \theta, q)>0$ such that for every $u \in D_{\mathrm{rad}}^{1, p}\left(\mathbb{R}^{N}\right)$, $h \in D^{1, p}\left(\mathbb{R}^{N}\right)$ and $R>0$ one has 


$$
\int_{B_{R}^{c}}|x|^{\theta}|u|^{q-1}|h| d x \leq \frac{C_{\infty}}{R^{\left(q-p^{*}-\frac{\theta p}{N-p}\right) \frac{N-p}{p}}}\|u\|_{D^{1, p}}^{q-1}\|h\|_{D^{1, p}} .
$$

Proof. We denote by $C$ any positive constant only depending on $N, p, \theta, q$. Taking $\delta=\delta(N, p, q)>0$ as in the previous lemma, the same computation giving (4.3) yields that for every $u \in D_{\text {rad }}^{1, p}\left(\mathbb{R}^{N}\right), h \in D^{1, p}\left(\mathbb{R}^{N}\right)$ and $R>0$ one has

$$
\begin{aligned}
& \int_{B_{R}^{c}}|x|^{\theta}|u|^{q-1}|h| d x \\
& \quad \leq C\left(\int_{R}^{+\infty} \rho^{\left(\theta-\frac{\delta N}{p^{*}}\right) \frac{p^{*}}{p^{*}-q+\delta}+N-1} d \rho\right)^{\left(p^{*}-q+\delta\right) / p^{*}}\|u\|_{D^{1, p}}^{q-1}\|h\|_{D^{1, p}}
\end{aligned}
$$

where

$$
\left(\theta-\frac{\delta N}{p^{*}}\right) \frac{p^{*}}{p^{*}-q+\delta}+N=\frac{N}{p^{*}-q+\delta}\left(p^{*}+\frac{\theta p}{N-p}-q\right)<0
$$

by assumption. Hence we obtain

$$
\int_{B_{R}^{c}}|x|^{\theta}|u|^{q-1}|h| d x \leq C\left(R^{\left(\theta-\frac{\delta N}{p^{*}}\right) \frac{p^{*}}{p^{*}-q+\delta}+N}\right)^{\left(p^{*}-q+\delta\right) / p^{*}}\|u\|_{D^{1, p}}^{q-1}\|h\|_{D^{1, p}},
$$

and the result then ensues.

Proof of Theorem 4.1. Let $u_{n} \rightarrow 0$ in $D_{\text {rad }}^{1, p}\left(\mathbb{R}^{N}\right)$, whence $\left\{u_{n}\right\}$ is bounded in $D^{1, p}\left(\mathbb{R}^{N}\right)$, and let $\varepsilon>0$. By assumption (4.2), there exist $C_{1}, C_{2}>0$ such that $\omega(x) \leq C_{1}|x|^{\theta_{1}}$ for almost every $|x|$ small enough and $\omega(x) \leq C_{2}|x|^{\theta_{2}}$ for almost every $|x|$ large enough, so that, by Lemmas 4.3 and 4.4 (applied with $u=h=u_{n}$ ), we can fix $r_{\varepsilon}, R_{\varepsilon}>0$ such that

$$
\begin{aligned}
& \int_{B_{r_{\varepsilon}}}\left|u_{n}\right|^{q_{1}} \omega(x) d x+\int_{B_{R_{\varepsilon}}^{c}}\left|u_{n}\right|^{q_{2}} \omega(x) d x \\
& \quad \leq C_{1} \int_{B_{r_{\varepsilon}}}|x|^{\theta_{1}}\left|u_{n}\right|^{q_{1}} d x+C_{2} \int_{B_{R_{\varepsilon}}^{c}}|x|^{\theta_{2}}\left|u_{n}\right|^{q_{2}} d x \\
& \leq C_{1} C_{0} r_{\varepsilon}^{\left(p^{*}+\frac{\theta p}{N-p}-q_{1}\right) \frac{N-p}{p}}\left\|u_{n}\right\|_{D^{1, p}}^{q_{1}}+\frac{C_{2} C_{\infty}}{R_{\varepsilon}^{\left(q_{2}-p^{*}-\frac{\theta p}{N-p}\right) \frac{N-p}{p}}}\left\|u_{n}\right\|_{D^{1, p}}^{q_{2}} \\
& \quad \leq C\left(r_{\varepsilon}^{\left(p^{*}+\frac{\theta p}{N-p}-q_{1}\right) \frac{N-p}{p}}+\frac{1}{R_{\varepsilon}^{\left(q_{2}-p^{*}-\frac{\theta p}{N-p}\right) \frac{N-p}{p}}}\right)<\frac{\varepsilon}{2}
\end{aligned}
$$

for some suitable constant $C>0$ and for all $n$. Then, if $q_{1} \leq p$, from the compactness of the embedding $D_{\text {rad }}^{1, p}\left(\mathbb{R}^{N}\right) \hookrightarrow L_{\text {loc }}^{p}\left(\mathbb{R}^{N}\right)$ we deduce that

$$
\int_{B_{R_{\varepsilon}} \backslash B_{r_{\varepsilon}}}\left|u_{n}\right|^{q_{1}} \omega(x) d x \leq\|\omega\|_{L^{\infty}\left(B_{R_{\varepsilon}} \backslash B_{r_{\varepsilon}}\right)} \int_{B_{R_{\varepsilon}} \backslash B_{r_{\varepsilon}}}\left|u_{n}\right|^{q_{1}} d x \rightarrow 0
$$


as $n \rightarrow \infty$. On the other hand, if $q_{1}>p$, we use Lemma 4.2 to deduce that there exists a constant $C_{\varepsilon}>0$ such that

$$
\begin{aligned}
& \int_{B_{R_{\varepsilon}} \backslash B_{r_{\varepsilon}}}\left|u_{n}\right|^{q_{1}} \omega(x) d x \\
& \leq\|\omega\|_{L^{\infty}\left(B_{R_{\varepsilon}} \backslash B_{r_{\varepsilon}}\right)} \int_{B_{R_{\varepsilon} \backslash B_{r_{\varepsilon}}}\left|u_{n}\right|^{q_{1}-p}\left|u_{n}\right|^{p} d x} \\
& \quad \leq\|\omega\|_{L^{\infty}\left(B_{R_{\varepsilon}} \backslash B_{r_{\varepsilon}}\right)} \frac{C_{N, p}^{q_{1}-p}\left\|u_{n}\right\|_{D^{1, p}\left(\mathbb{R}^{N}\right)}^{q_{1}-p}}{r_{\varepsilon}^{\frac{N-p}{p}\left(q_{1}-p\right)}} \int_{B_{R_{\varepsilon} \backslash B_{r_{\varepsilon}}}\left|u_{n}\right|^{p} d x} \int^{\leq} C_{\varepsilon} \int_{B_{R_{\varepsilon} \backslash B_{r_{\varepsilon}}}\left|u_{n}\right|^{p} d x \rightarrow 0}
\end{aligned}
$$

as $n \rightarrow \infty$. Therefore condition (2.7) holds with $E_{\varepsilon, n}=B_{R_{\varepsilon}}$ and the conclusion follows from Proposition 2.7.

The following results are straightforward consequences of Theorem 4.1. In particular, the first one contains a compactness lemma due to Benci-Fortunato (see [15, Lemma 3]), corresponding to the case $p=2$ and $\omega$ constant.

Corollary 4.5. If $\omega \in L^{\infty}\left(\mathbb{R}^{N}\right)$, then the space $D_{\mathrm{rad}}^{1, p}\left(\mathbb{R}^{N}\right)$ is compactly embedded into $L\left(\mathbb{R}^{N}, \omega(x) d x\right)$ for every $1<q_{1}<p^{*}<q_{2}$.

Proof. If $\omega \in L^{\infty}\left(\mathbb{R}^{N}\right)$ then condition (4.2) holds with $\theta_{1}=\theta_{2}=0$ (which are also the best exponents for such a condition) and the result follows from Theorem 4.1 .

Corollary 4.6. If $\omega \in L_{\mathrm{loc}}^{\infty}\left(\mathbb{R}^{N} \backslash\{0\}\right)$ and condition (4.2) holds for some $\theta_{1}>$ $-\left(1+N / p^{\prime}\right)$ and $\theta_{2}<\theta_{1}$, then $D_{\text {rad }}^{1, p}\left(\mathbb{R}^{N}\right)$ is compactly embedded into $L^{q}$ $\left(\mathbb{R}^{N}, \omega(x) d x\right)$ for every $q>1$ such that

$$
p^{*}+\frac{\theta_{2} p}{N-p}<q<p^{*}+\frac{\theta_{1} p}{N-p} .
$$

Proof. Since $\theta_{2}<\theta_{1}$ implies $p^{*}+\theta_{2} p /(N-p)<p^{*}+\theta_{1} p /(N-p)$, we can apply Theorem 4.1 with $q_{1}=q_{2}=q$ and the result ensues, because $L\left(\mathbb{R}^{N}, \omega(x) d x\right)=$ $L^{q}\left(\mathbb{R}^{N}, \omega(x) d x\right)$.

\section{Application to quasilinear equations}

Assume $1<p<N$ and let $V:(0,+\infty) \rightarrow[0,+\infty]$ and $f:(0,+\infty) \times \mathbb{R} \rightarrow$ $[0,+\infty)$ be, respectively, a measurable and a Carathéodory function satisfying $(\mathbf{V})$ and $(\mathbf{f})$.

We define the weighted Sobolev spaces

$$
\begin{aligned}
W & =W^{1, p}\left(\mathbb{R}^{N}, V\right):=\left\{u \in D^{1, p}\left(\mathbb{R}^{N}\right): \int_{\mathbb{R}^{N}} V(|x|)|u|^{p} d x<+\infty\right\}, \\
W_{\mathrm{rad}} & =W_{\mathrm{rad}}^{1, p}\left(\mathbb{R}^{N}, V\right):=\overline{C_{\mathrm{c}, \mathrm{rad}}^{\infty}\left(\mathbb{R}^{N}\right) \cap W^{1, p}\left(\mathbb{R}^{N}, V\right)} W^{1, p}\left(\mathbb{R}^{N}, V\right)
\end{aligned}
$$


equipped with the norm given by

$$
\|u\|_{W}^{p}:=\int_{\mathbb{R}^{N}}\left(|\nabla u|^{p}+V(|x|)|u|^{p}\right) d x,
$$

with respect to which they are uniformly convex Banach spaces. Note that assumption ( V) ensures that both $W$ and $W_{\text {rad }}$ are nonempty. Moreover, since $W$-convergence implies almost everywhere convergence (up to a subsequence), all the mappings in $W_{\text {rad }}$ are spherically symmetric.

We will apply some results from the previous sections (in particular Theorem 4.1, Proposition 3.8 and Corollary 2.19) with $\Omega=\mathbb{R}^{N}$ and $d \mu=\omega(x) d x$, where

$$
\omega(x)=\max \left\{|x|^{\theta_{1}},|x|^{\theta_{2}}\right\}
$$

according to assumption (f). Note that $W_{\text {rad }}$ is continuously embedded into $D_{\text {rad }}^{1, p}\left(\mathbb{R}^{N}\right)$ and thus the embedding

$$
W_{\mathrm{rad}} \hookrightarrow L\left(\mathbb{R}^{N}, \omega(x) d x\right):=L^{q_{1}}\left(\mathbb{R}^{N}, \omega(x) d x\right)+L^{q_{2}}\left(\mathbb{R}^{N}, \omega(x) d x\right)
$$

(where $q_{1}, q_{2}$ are given by (f) again) is compact by Theorem 4.1 .

Thanks to the continuity of the embedding $W_{\text {rad }} \hookrightarrow L\left(\mathbb{R}^{N}, \omega(x) d x\right)$, assumption (f) and Proposition 3.8 ensure that the functional $I: W_{\mathrm{rad}} \rightarrow \mathbb{R}$ given by

$$
I(u):=\frac{1}{p}\|u\|_{W}^{p}-\int_{\mathbb{R}^{N}} F(|x|, u) d x
$$

(where $\left.F(r, t):=\int_{0}^{t} f(r, s) d s\right)$ is well defined and of class $C^{1}$, with Fréchet derivative $I^{\prime}(u) \in W_{\text {rad }}^{\prime}$ at any $u \in W_{\text {rad }}$ given by

$$
\left\langle I^{\prime}(u), h\right\rangle=\int_{\mathbb{R}^{N}}\left(|\nabla u|^{p-2} \nabla u \cdot \nabla h+V(|x|)|u|^{p-2} u h-f(|x|, u) h\right) d x
$$

for all $h \in W_{\text {rad. }}$. Hence the critical points $u \in W_{\text {rad }}$ of $I$ satisfy (1.3) for all $h \in W_{\text {rad }}$. The next lemma shows that $W_{\text {rad }}$ actually is, in some sense, a natural constraint for finding weak solutions of equation (1.2). Observe that the classical Palais' principle of symmetric criticality [32] does not apply in this case, because we do not know whether $I$ is differentiable, not even well defined, on the whole space $W$ or not.

Lemma 5.1. Every critical point of $I: W_{\mathrm{rad}} \rightarrow \mathbb{R}$ is a weak solution to equation (1.2).

Proof. We show that if $u \in W_{\text {rad }}$ satisfies (1.3) for all $h \in W_{\text {rad }}$, then (1.3) holds also true for all $h \in W$. Let $u \in W_{\text {rad }}$. By (f) and Lemmas 4.3 and 4.4, $\forall h \in W$ we have

$$
\begin{aligned}
\int_{\mathbb{R}^{N}}|f(|x|, u) h| d x & \leq M \int_{\mathbb{R}^{N}} \min \left\{|u|^{q_{1}-1},|u|^{q_{2}-1}\right\}|h| \omega(x) d x \\
& \leq M \int_{B_{1}}|x|^{\theta_{1}}|u|^{q_{1}-1}|h| d x+M \int_{B_{1}^{c}}|x|^{\theta_{2}}|u|^{q_{2}-1}|h| d x \\
& \leq M\left(C_{0}\|u\|_{W}^{q_{1}-1}+C_{\infty}\|u\|_{W}^{q_{2}-1}\right)\|h\|_{W},
\end{aligned}
$$


so that the linear functional defined by

$$
\langle T(u), h\rangle:=\int_{\mathbb{R}^{N}}\left(|\nabla u|^{p-2} \nabla u \cdot \nabla h+V(|x|)|u|^{p-2} u h-f(|x|, u) h\right) d x
$$

is well defined and continuous on $W$. Hence, by uniform convexity, there exists a unique $\tilde{u} \in W$ such that $T(u) \tilde{u}=\|\tilde{u}\|_{W}^{2}=\|T(u)\|_{W^{\prime}}^{2}$. Then, by means of obvious changes of variable, one checks that for every $h \in W$ we have

$$
\langle T(u), h(S \cdot)\rangle=\langle T(u), h\rangle \quad \text { and } \quad\|h(S \cdot)\|_{W}=\|h\|_{W} \quad \text { for all } S \in O(N),
$$

whence, applying with $h=\tilde{u}$, one deduces $\tilde{u}(S \cdot)=\tilde{u}$ by uniqueness. This means $\tilde{u} \in W_{\text {rad }}$, so that, if $\langle T(u), h\rangle=0$ for all $h \in W_{\text {rad, one has }}$ $\langle T(u), \tilde{u}\rangle=0$, that is, $\|T(u)\|_{W^{\prime}}=0$.

By virtue of Lemma 5.1, the proof of Theorems 1.1 and 1.2 reduces to finding critical points of the functional $I$, which exhibits a right amount of compactness, according to the following lemma.

Lemma 5.2. The functional $I: W_{\mathrm{rad}} \rightarrow \mathbb{R}$ satisfies the Palais-Smale condition.

Proof. Let $\left\{u_{n}\right\} \subseteq W_{\text {rad }}$ be a sequence such that $\left\{I\left(u_{n}\right)\right\}$ is bounded and $I^{\prime}\left(u_{n}\right) \rightarrow 0$ in $W_{\text {rad }}^{\prime}$. One has to show that $\left\{u_{n}\right\}$ contains a $W$-converging subsequence. Exploiting the condition $\gamma F(r, t) \leq f(r, t) t$ with $\gamma>p$ of assumption (f), a standard argument shows that $\left\{u_{n}\right\}$ is bounded in $W_{\text {rad }}$. Then Theorem 4.1 applies, yielding the existence of $u \in W_{\text {rad }}$ such that (up to a subsequence)

$$
\begin{aligned}
& u_{n} \rightarrow u \text { in } W_{\mathrm{rad}} \\
& u_{n} \rightarrow u \text { in } L\left(\mathbb{R}^{N}, \omega(x) d x\right) .
\end{aligned}
$$

Now set

$$
I_{1}(u):=\frac{1}{p}\|u\|_{W}^{p} \quad \text { and } \quad I_{2}(u):=I_{1}(u)-I(u)
$$

for brevity. Then, by (5.1) and Proposition 3.8, we get

$$
\left\|u_{n}\right\|_{W}^{p}=\left\langle I^{\prime}\left(u_{n}\right), u_{n}\right\rangle+\left\langle I_{2}^{\prime}\left(u_{n}\right), u_{n}\right\rangle=\left\langle I_{2}^{\prime}(u), u\right\rangle+o(1)_{n \rightarrow \infty},
$$

so that $\lim _{n \rightarrow \infty}\left\|u_{n}\right\|_{W}$ exists and one has $\|u\|_{W}^{p} \leq \lim _{n \rightarrow \infty}\left\|u_{n}\right\|_{W}^{p}$ by weak lower semicontinuity. Moreover the convexity of $I_{1}: W_{\text {rad }} \rightarrow \mathbb{R}$ implies

$$
\begin{aligned}
I_{1}(u)-I_{1}\left(u_{n}\right) & \geq\left\langle I_{1}^{\prime}\left(u_{n}\right), u-u_{n}\right\rangle \\
& =\left\langle I^{\prime}\left(u_{n}\right), u-u_{n}\right\rangle+\left\langle I_{2}^{\prime}\left(u_{n}\right), u-u_{n}\right\rangle=o(1)_{n \rightarrow \infty}
\end{aligned}
$$

and thus

$$
\frac{1}{p}\|u\|_{W}^{p}=I_{1}(u) \geq \lim _{n \rightarrow \infty} I_{1}\left(u_{n}\right)=\frac{1}{p} \lim _{n \rightarrow \infty}\left\|u_{n}\right\|_{W}^{p} .
$$

So $\left\|u_{n}\right\|_{W} \rightarrow\|u\|_{W}$ and one concludes that $u_{n} \rightarrow u$ in $W_{\text {rad }}$ by uniform convexity. 
Proof of Theorem 1.1. As we are interested in nonnegative solutions, it is not restrictive to assume

$$
f(r, t)=0 \quad \text { for all } r>0 \text { and } t<0 .
$$

We want to apply the well known Mountain-Pass Theorem [2]. To this end, observe that, by (f) and Lemmas 4.3 and 4.4 (applied with $h=u$ ), for every $u \in W_{\text {rad }}$ we have

$$
\begin{aligned}
\int_{\mathbb{R}^{N}} F(|x|, u) d x & \leq c_{1} \int_{\mathbb{R}^{N}} \min \left\{|u|^{q_{1}},|u|^{q_{2}}\right\} \omega(x) d x \\
& \leq \int_{B_{1}}|u|^{q_{1}}|x|^{\theta_{1}} d x+\int_{B_{1}^{c}}|u|^{q_{2}}|x|^{\theta_{2}} d x \\
& \leq c_{2}\|u\|_{W}^{q_{1}}+c_{3}\|u\|_{W}^{q_{2}}
\end{aligned}
$$

(where $c_{1}, c_{2}, c_{3}$ denote some positive constants), so that

$$
I(u) \geq \frac{1}{p}\|u\|_{W}^{p}-c_{2}\|u\|_{W}^{q_{1}}-c_{3}\|u\|_{W}^{q_{2}} .
$$

Since $q_{2}>q_{1}>p$, this proves that $I$ has a mountain pass geometry near the origin, i.e., there exist $\delta, \rho>0$ such that for all $u \in W_{\text {rad }}$ with $\|u\|_{W}=\rho$ one has $I(u) \geq \delta$. On the other hand, there exists $\bar{u} \in W_{\text {rad }}$ such that $\|\bar{u}\|_{W}>\rho$ and $I(\bar{u})<0$. Indeed, by assumption (1.7) and condition $\gamma F(r, t) \leq f(r, t) t$ of $(\mathbf{f})$, one easily deduces

$$
F(r, t) \geq \frac{F\left(r, t_{*}\right)}{t_{*}^{\gamma}} t^{\gamma} \quad \text { for almost every } r>0 \quad \text { and all } t \geq t_{*}
$$

so that, $\forall \lambda>1$ and $\forall u \in W_{\text {rad }}$ nonnegative such that the set $\left\{x \in \mathbb{R}^{N}\right.$ : $\left.u(x) \geq t_{*}\right\}$ has positive Lebesgue measure, we get

$$
\begin{aligned}
\int_{\mathbb{R}^{N}} F(|x|, \lambda u) d x & \geq \int_{\left\{\lambda u \geq t_{*}\right\}} F(|x|, \lambda u) d x \geq \frac{\lambda^{\gamma}}{t_{*}^{\gamma}} \int_{\left\{\lambda u \geq t_{*}\right\}} F\left(|x|, t_{*}\right) u^{\gamma} d x \\
& \geq \frac{\lambda^{\gamma}}{t_{*}^{\gamma}} \int_{\left\{u \geq t_{*}\right\}} F\left(|x|, t_{*}\right) u^{\gamma} d x \\
& \geq \lambda^{\gamma} \int_{\left\{u \geq t_{*}\right\}} F\left(|x|, t_{*}\right) d x>0
\end{aligned}
$$

(recall that $F \geq 0$ and $F\left(|\cdot|, t_{*}\right)>0$ almost everywhere), which gives

$$
I(\lambda u) \leq \frac{\lambda^{p}}{p}\|u\|_{W}^{p}-\lambda^{\gamma} \int_{\left\{u \geq t_{*}\right\}} F\left(|x|, t_{*}\right) d x \rightarrow-\infty \quad \text { as } \lambda \rightarrow+\infty
$$

since $\gamma>p$. As a conclusion, $I$ exhibits a full mountain-pass geometry and, by Lemma 5.2, the Mountain-Pass Theorem provides the existence of a nontrivial critical point for $I$, which is a weak solution to equation (1.2) by Lemma 5.1. Finally, by (5.2), a standard argument shows that any $u \in W$ satisfying (1.3) for all $h \in W$ has to be nonnegative.

Proof of Theorem 1.2. By the oddness assumption (1.8), one has $I(u)=$ $I(-u)$ for all $u \in W_{\text {rad }}$ and thus we can apply the Symmetric MountainPass Theorem (see for example [36, Theorem 6.5]). To this end, taking into 
account (5.3) and Lemma 5.2, we need only to show that $I$ satisfies the following geometrical condition: for any finite dimensional subspace $Y \neq\{0\}$ of $W_{\text {rad }}$ there exists $R>0$ such that for all $u \in Y$ with $\|u\|_{W} \geq R$ one has $I(u) \leq 0$. In fact it is sufficient to prove that any sequence $\left\{u_{n}\right\} \subseteq Y$ with $\left\|u_{n}\right\|_{W} \rightarrow$ $+\infty$ admits a subsequence such that $I\left(u_{n}\right) \leq 0$. Recall the definition (2.1) (where $\Omega=\mathbb{R}^{N}$ ) of $\Lambda_{u_{n}}$ and briefly denote $L^{q_{1}}\left(\Lambda_{u_{n}}\right):=L^{q_{1}}\left(\Lambda_{u_{n}}, \omega(x) d x\right)$, $L^{q_{2}}\left(\Lambda_{u_{n}}^{c}\right):=L^{q_{2}}\left(\Lambda_{u_{n}}^{c}, \omega(x) d x\right)$. Since all norms are equivalent on $Y$, one has

$$
\left\|u_{n}\right\|_{L^{q_{1}\left(\Lambda_{u_{n}}\right)}}+\left\|u_{n}\right\|_{L^{q_{2}\left(\Lambda_{u_{n}}^{c}\right)}} \geq\left\|u_{n}\right\| \geq c_{0}\left\|u_{n}\right\|_{W} \rightarrow+\infty
$$

for some constant $c_{0}>0$, where the right hand inequality of (2.21) has been used. Thus, up to a subsequence, at least one of the sequences $\left\{\left\|u_{n}\right\|_{L^{q_{1}\left(\Lambda_{u_{n}}\right)}}\right\}$, $\left\{\left\|u_{n}\right\|_{L^{q_{2}\left(\Lambda_{u_{n}}^{c}\right)}}\right\}$ diverges. We now use assumption (1.9) to obtain

$$
\int_{\mathbb{R}^{N}} F\left(|x|, u_{n}\right) d x \geq m \int_{\Lambda_{u_{n}}}\left|u_{n}\right|^{q_{1}} \omega(x) d x+m \int_{\Lambda_{u_{n}}^{c}}\left|u_{n}\right|^{q_{2}} \omega(x) d x .
$$

Hence, using inequalities (5.4), there exists a constant $c>0$ such that

$$
\begin{aligned}
& I\left(u_{n}\right) \\
& \quad \leq c\left(\left\|u_{n}\right\|_{L^{q_{1}\left(\Lambda_{u_{n}}\right)}}^{p}+\left\|u_{n}\right\|_{L^{q_{2}\left(\Lambda_{u_{n}}^{c}\right)}}^{p}\right)-m\left(\left\|u_{n}\right\|_{L^{q_{1}\left(\Lambda_{u_{n}}\right)}}^{q_{1}}+\left\|u_{n}\right\|_{L^{q_{2}\left(\Lambda_{u_{n}}^{c}\right)}}^{q_{2}}\right) \\
& \quad=c\left\|u_{n}\right\|_{L^{q_{1}\left(\Lambda_{u_{n}}\right)}}^{p}-m\left\|u_{n}\right\|_{L^{q_{1}\left(\Lambda_{u_{n}}\right)}}^{q_{1}}+c\left\|u_{n}\right\|_{L^{q_{2}\left(\Lambda_{u_{n}}^{c}\right)}}^{p}-m\left\|u_{n}\right\|_{L^{q_{2}\left(\Lambda_{u_{n}}^{c}\right)}}^{q_{2}},
\end{aligned}
$$

so that $I\left(u_{n}\right) \rightarrow-\infty$, since $q_{2}>q_{1}>p$. Therefore the Symmetric MountainPass Theorem implies the existence of an unbounded sequence of critical values for $I$, to which there corresponds a sequence of nontrivial critical points and thus a sequence of weak solutions to equation (1.2), thanks to Lemma 5.1.

\section{References}

[1] Ambrosetti, A., Felli, V., Malchiodi, A.: Ground states of nonlinear Schrödinger equations with potentials vanishing at infinity. J. Eur. Math. Soc. 7, 117$144(2005)$

[2] Ambrosetti, A., Rabinowitz, P.H.: Dual variational methods in critical point theory and applications. J. Funct. Anal. 14, 349-381 (1973)

[3] Ambrosetti, A., Wang, Z.Q.: Nonlinear Schrödinger equations with vanishing and decaying potentials. Differ. Int. Equ. 18, 1321-1332 (2005)

[4] Azzollini, A.: A multiplicity result for a semilinear Maxwell type equation. Topol. Methods Nonlinear Anal. 31, 83-110 (2008)

[5] Azzollini, A., Pisani, L., Pomponio, A.: Improved estimates and a limit case for the electrostatic Klein-Gordon-Maxwell system. Proc. R. Soc. Edinb. (2010, in press)

[6] Azzollini, A., Pomponio, A.: On a "zero mass" nonlinear Schrödinger equation. Adv. Nonlinear Stud. 7, 599-627 (2007) 
[7] Azzollini, A., Pomponio, A.: Compactness results and applications to some "zero mass" elliptic problems. Nonlinear Anal. 69, 3559-3576 (2008)

[8] Badiale, M., Benci, V., Rolando, S.: A nonlinear elliptic equation with singular potential and applications to nonlinear field equations. J. Eur. Math. Soc. 9, 355$381(2007)$

[9] Badiale, M., Benci, V., Rolando, S.: Three dimensional vortices in the nonlinear wave equation. Bollettino U.M.I. Serie IX 2, 105-134 (2009)

[10] Badiale, M., Guida, M., Rolando, S.: Elliptic equations with decaying cylindrical potentials and power-type nonlinearities. Adv. Differ. Equ. 12, 1321-1362 (2007)

[11] Badiale, M., Rolando, S.: Elliptic problems with singular potentials and doublepower nonlinearity. Mediterr. J. Math. 2, 417-436 (2005)

[12] Badiale, M., Rolando, S.: A note on nonlinear elliptic problems with singular potentials. Rend. Lincei Mat. Appl. 16, 1-13 (2006)

[13] Badiale, M., Rolando, S.: Nonlinear elliptic equations with subhomogeneous potentials. Nonlinear Anal. 72, 602-617 (2010)

[14] Benci, V., Fortunato, D.: A strongly degenerate elliptic equation arising from the semilinear Maxwell equations. C. R. Acad. Sci. Paris série I 339, 839-842 (2004)

[15] Benci, V., Fortunato, D.: Towards a unified field theory for classical electrodynamics. Arch. Ration. Mech. Anal. 173, 379-414 (2004)

[16] Benci, V., Grisanti, C.R., Micheletti, A.M.: Existence and non existence of the ground state solution for the nonlinear Schrödinger equation with $V(\infty)=$ 0. Topol. Methods Nonlinear Anal. 26, 203-220 (2005)

[17] Benci, V., Grisanti, C.R., Micheletti, A.M.: Existence of solutions for the nonlinear Schrödinger equation with $V(\infty)=0$. In: Progress in Nonlinear Differential Equations and Their Applications, vol. 66. Birkhäuser (2005)

[18] Benci, V., Micheletti, A.M.: Solutions in exterior domains of null mass nonlinear field equations. Adv. Nonlinear Stud. 6, 171-198 (2006)

[19] Berestycki, H., Lions, P.L.: Nonlinear scalar field equations, I- existence of a ground state. Arch. Ration. Mech. Anal. 82, 313-345 (1983)

[20] Berestycki, H., Lions, P.L.: Existence d'états multiples dans des équations de champs scalaires non linéaires dans le cas de masse nulle. C. R. Acad. Sci. Paris série I 297, 267-270 (1983)

[21] Bonheure, D.,Van Schaftingen, J.: Bound states solutions for a class of nonlinear Schrödinger equations. Rev. Mat. Iberoamericana 24, 297-351 (2008)

[22] Brezis, H., Dupaigne, L., Tesei, A.: On a semilinear elliptic equation with inversesquare potential. Selecta Math. New Series 11, 1-7 (2005)

[23] Byeon, J., Wang, Z.Q.: Standing waves with a critical frequency for nonlinear Schrödinger equations. Arch. Ration. Mech. Anal. 165, 295-316 (2002) 
[24] Byeon, J., Wang, Z.Q.: Standing waves with a critical frequency for nonlinear Schrödinger equations. II. Calc. Var. Partial Differ. Equ. 18, 207-219 (2003)

[25] Byeon, J., Wang, Z.Q.: Spherical semiclassical states of a critical frequency for Schrödinger equations with decaying potentials. J. Eur. Math. Soc. 8, 217$228(2006)$

[26] Conti, M., Crotti, S., Pardo, D.: On the existence of positive solutions for a class of singular elliptic equations. Adv. Differ. Equ. 3, 111-132 (1998)

[27] Drewnowski, L., Orlicz, W.: A note on modular spaces. XI. Bull. Acad. Polon. Sci. Ser. Sci. Math. Astronom. Phys. 16, 877-882 (1968)

[28] Ghimenti, M., Micheletti, A.M.: Solutions for a nonhomogeneous nonlinear Schroedinger equation with double-power nonlinearity. Differ. Int. Equ. 10, 1131-1152 (2007)

[29] Krasnosel'skii, M.A., Rutickii, Y.B.: Convex Functions and Orlicz Spaces. P. Noordhoff Ltd, Groningen (1961)

[30] Maligranda, L.: Orlicz spaces and interpolation. In: Seminars in Mathematics, vol. 5. Univ. Estadual de Campinas, Campinas (1989)

[31] Musina, R.: Existence and multiplicity results for a weighted $p$-Laplace equation involving Hardy potentials and critical nonlinearities. Rend. Lincei Mat. Appl. 20, 1-17 (2009)

[32] Palais, R.S.: The principle of symmetric criticality. Commun. Math. Phys. 69, 19-30 (1979)

[33] Pisani, L.: Remarks on the sum of Lebesgue spaces. Unpublished note (2004)

[34] Rao, M.M., Ren, Z.D.: Theory of Orlicz Spaces. Marcel Dekker, New York (1991)

[35] Rolando, S.: Nonlinear elliptic equations with singular symmetric potentials. $\mathrm{PhD}$ Thesis, Dipartimento di Matematica, Università degli Studi di Torino (2006). http://www2.dm.unito.it/paginepersonali/rolando

[36] Struwe, M.: Variational Methods. Springer-Verlag, Berlin-Heidelberg (1990)

[37] Su, J., Wang, Z.Q., Willem, M.: Weighted Sobolev embedding with unbounded and decaying radial potentials. J. Differ. Equ. 238, 201-219 (2007)

[38] Vainberg, M.M.: Variational Methods for the Study of Nonlinear Operators. Holden-Day, San Francisco (1964)

Marino Badiale

Dipartimento di Matematica,

Università degli Studi di Torino,

Via Carlo Alberto 10,

10123 Turin,

Italy

e-mail: marino.badiale@unito.it 


\section{Lorenzo Pisani}

Dipartimento di Matematica,

Università degli Studi di Bari,

Via Orabona 4,

70125 Bari,

Italy

e-mail: pisani.dm.uniba@gmail.com

Sergio Rolando

Dipartimento di Matematica,

Università degli Studi di Torino,

Via Carlo Alberto 10,

10123 Turin,

Italy

e-mail: sergio.rolando@unito.it

Received: 24 August 2010.

Accepted: 06 January 2011. 